\title{
Cascade Coupling/Cyclization Process to N-Substituted
}

\section{1,3-Dihydrobenzimidazol-2-ones}

\author{
Benli Zou, ${ }^{1}$ Qiliang Yuan, ${ }^{1}$ and Dawei $\mathrm{Ma}^{2} *$ \\ ${ }^{1}$ Department of Chemistry, Fudan University, Shanghai 200433, China \\ ${ }^{2}$ State Key Laboratory of Bioorganic and Natural Products Chemistry, Shanghai Institute of \\ Organic Chemistry, Chinese Academy of Sciences, 354 Fenglin Lu, Shanghai 200032, China
}

\section{Supporting Information}

\section{Table of Contents}

Experimental-

S2-S9

Copies of ${ }^{1} \mathrm{H}$ NMR and ${ }^{13} \mathrm{C}$ NMR spectrum of $\mathbf{8 a - 8} \mathbf{q}$

$-S 10-S 43$ 


\section{Experimental}

General procedure for synthesis of benzimidazol-2-ones from aryl bromides: A Schlenk tube was charged with aryl bromide $(0.5 \mathrm{mmol}), \mathrm{CuI}(19 \mathrm{mg}, 0.1 \mathrm{mmol})$, trans-4-hydro-L-proline (26 mg, $0.2 \mathrm{mmol})$, and $\mathrm{K}_{3} \mathrm{PO}_{4}(212 \mathrm{mg}, 1.0 \mathrm{mmol})$, evacuated and backfilled with argon. Amine $(0.5 \mathrm{mmol})$ and DMSO $(1 \mathrm{~mL})$ were successively added. The reaction mixture was stirred at 70 ${ }^{\circ} \mathrm{C}$ until the coupling was completed detected by TLC. The solution was then heated at $130{ }^{\circ} \mathrm{C}$ until the coupling product was consumed monitored by TLC. The cold mixture was partitioned between ethyl acetate and saturated $\mathrm{NH}_{4} \mathrm{Cl}$. The organic layer was washed with brine, dried over $\mathrm{Na}_{2} \mathrm{SO}_{4}$ and concentrated in vacuo. The residue was purified by column chromatography on silica gel to provide the desired product.<smiles>O=c1[nH]c2ccccc2n1Cc1ccccc1</smiles>

1-Benzyl-1H-benzo[d]imidazol-2(3H)-one 8a: ${ }^{1} \mathrm{H}$ NMR $\left(400 \mathrm{MHz}, \mathrm{CDCl}_{3}\right) \delta 9.45(\mathrm{~s}, 1 \mathrm{H})$, $7.32(\mathrm{~m}, 5 \mathrm{H}), 7.10-6.88(\mathrm{~m}, 4 \mathrm{H}), 5.09(\mathrm{~s}, 2 \mathrm{H}) ;{ }^{13} \mathrm{C} \mathrm{NMR}\left(100 \mathrm{MHz}, \mathrm{CDCl}_{3}\right) \delta 155.8,139.2,136.2$ 130.2, 128.8, 127.8, 128.1, 127.5, 127.4, 121.8, 121.4, 109.8, 108.6, 44.5; ESI-MS m/z 225.2 (M + $\mathrm{H})^{+}$; ESI-HRMS calcd. for $\mathrm{C}_{14} \mathrm{H}_{12} \mathrm{~N}_{2} \mathrm{O}\left(\mathrm{M}^{+}\right)$requires 224.0950, found 224.0958.<smiles>O=C(O)Cn1c(=O)[nH]c2ccccc21</smiles>

2-(2-Oxo-2,3-dihydro-1H-benzo[d]imidazol-1-yl)acetic acid 8b: ${ }^{1} \mathrm{H}$ NMR (400 MHz, $\left.\mathrm{CD}_{3} \mathrm{SOCD}_{3}\right) \delta 10.92($ br s, $1 \mathrm{H}), 7.35-6.81(\mathrm{~m}, 4 \mathrm{H}), 4.11(\mathrm{~s}, 2 \mathrm{H}) ;{ }^{13} \mathrm{C} \mathrm{NMR}\left(100 \mathrm{MHz}, \mathrm{CD}_{3} \mathrm{SOCD}_{3}\right)$ $\delta 171.9,155.2,131.8,128.9,120.6,120.5,108.9,108.6$, 44.7; ESI-MS $m / z 193.1(\mathrm{M}+\mathrm{H})^{+}$; ESI-HRMS calcd. for $\mathrm{C}_{7} \mathrm{H}_{5} \mathrm{~N}_{2} \mathrm{O}\left(\mathrm{M}-\mathrm{CH}_{2} \mathrm{CO}_{2} \mathrm{H}\right)^{+}$required 133.0402, found 133.0413. 


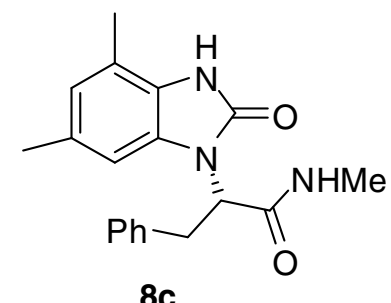

(S)-2-(4,6-Dimethyl-2-oxo-2,3-dihydrobenzo[d]imidazol-1-yl)- $N$-methyl-3-phenyl

propanamide 8c: ${ }^{1} \mathrm{H}$ NMR $\left(400 \mathrm{MHz}, \mathrm{CD}_{3} \mathrm{SOCD}_{3}\right) \delta 10.64$ (br s, $\left.1 \mathrm{H}\right), 7.93(\mathrm{~d}, J=4.6 \mathrm{~Hz}, 1 \mathrm{H})$, 7.15-7.02 (m, 3H), $6.97(\mathrm{~d}, J=7.3 \mathrm{~Hz}, 2 \mathrm{H}), 6.77(\mathrm{~s}, 1 \mathrm{H}), 6.55(\mathrm{~s}, 1 \mathrm{H}), 5.06(\mathrm{~m}, 1 \mathrm{H}), 334(\mathrm{~m}, 2 \mathrm{H})$, $2.55(\mathrm{~d}, J=4.1 \mathrm{~Hz}, 3 \mathrm{H}), 2.23(\mathrm{~s}, 3 \mathrm{H}), 2.15(\mathrm{~s}, 3 \mathrm{H}) ;{ }^{13} \mathrm{C} \mathrm{NMR}\left(100 \mathrm{MHz}, \mathrm{CD}_{3} \mathrm{SOCD}_{3}\right) \delta 169.47$, $154.98,138.27,129.61129 .27,128.58,126.75,125.53,123.27,118.34,108.21,56.44,34.39,26.51$, 21.69, 16.57; ESI-MS m/z $324.2(\mathrm{M}+\mathrm{H})^{+}$; ESI-HRMS calcd. for $\mathrm{C}_{19} \mathrm{H}_{22} \mathrm{~N}_{3} \mathrm{O}_{2}(\mathrm{M}+\mathrm{H})^{+}$required 324.1697, found 324.1707.<smiles>C=CCn1c(=O)[nH]c2ccc(OC)cc21</smiles>

1-Allyl-6-methoxy-1H-benzo[d]imidazol-2(3H)-one 8d: ${ }^{1} \mathrm{H}$ NMR $\left(400 \mathrm{MHz}, \mathrm{CDCl}_{3}\right) \delta$ 10.47 (br s, 1H), $7.02(\mathrm{~d}, J=8.3 \mathrm{~Hz}, 1 \mathrm{H}), 6.63(\mathrm{dd}, J=8.3,2.3 \mathrm{~Hz}, 1 \mathrm{H}), 6.57(\mathrm{~s}, 1 \mathrm{H}), 5.92(\mathrm{~m}, 1 \mathrm{H})$, $5.23(\mathrm{~m}, 2 \mathrm{H}), 4.50(\mathrm{dd}, J=5.0,1.4 \mathrm{~Hz}, 2 \mathrm{H}), 3.80(\mathrm{~s}, 3 \mathrm{H}) ;{ }^{13} \mathrm{C} \mathrm{NMR}\left(100 \mathrm{MHz}, \mathrm{CDCl}_{3}\right) \delta 156.23$, 155.38, 131.86, 131.14, 122.21, 117.55, 110.11, 106.94, 95.82, 56.02, 43.20. ESI-MS m/z 205.1 (M $+\mathrm{H})^{+}$; ESI-HRMS calcd. for $\mathrm{C}_{11} \mathrm{H}_{13} \mathrm{~N}_{2} \mathrm{O}_{2}(\mathrm{M}+\mathrm{H})^{+}$required 205.0973, found 205.0972.<smiles>CCCCCCn1c(=O)[nH]c2ccc(C(C)=O)cc21</smiles>

6-Acetyl-1-hexyl-1H-benzo[d]imidazol-2(3H)-one 8e: ${ }^{1} \mathrm{H}$ NMR $\left(400 \mathrm{MHz}, \mathrm{CDCl}_{3}\right) \delta$ 
$11.09($ br s, 1H), $7.75(\mathrm{dd}, J=8.2,1.4 \mathrm{~Hz}, 1 \mathrm{H}), 7.68(\mathrm{~s}, 1 \mathrm{H}), 7.19(\mathrm{~d}, J=8.2 \mathrm{~Hz}, 1 \mathrm{H}), 3.94(\mathrm{t}, J=$ $7.3 \mathrm{~Hz}, 2 \mathrm{H}), 2.64(\mathrm{~s}, 3 \mathrm{H}), 1.78(\mathrm{~m}, 2 \mathrm{H}), 1.32(\mathrm{~m}, 6 \mathrm{H}), 0.88(\mathrm{t}, J=6.9 \mathrm{~Hz}, 3 \mathrm{H}) ;{ }^{13} \mathrm{C} \mathrm{NMR}(100 \mathrm{MHz}$, $\left.\mathrm{CDCl}_{3}\right) \delta 197.37,156.39,132.54,131.19,130.68,123.85,109.08,107.50,41.20,31.51,28.49$, 26.63, 26.57, 22.63, 14.10; ESI-MS m/z $261.2(\mathrm{M}+\mathrm{H})^{+}$; ESI-HRMS calcd. for $\mathrm{C}_{15} \mathrm{H}_{21} \mathrm{~N}_{2} \mathrm{O}_{2}(\mathrm{M}+$ $\mathrm{H})^{+}$required 261.1611, found 261.1598.

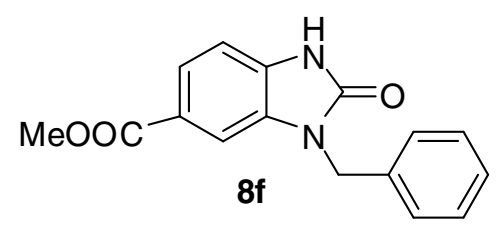

Methyl 3-benzyl-2-oxo-2,3-dihydro-1H-benzo[d]imidazole-5-carboxylate 8f: ${ }^{1} \mathrm{H}$ NMR $\left(400 \mathrm{MHz}, \mathrm{CDCl}_{3}\right) \delta 9.22(\mathrm{br} \mathrm{s}, 1 \mathrm{H}), 7.82(\mathrm{dd}, J=8.2,1.4 \mathrm{~Hz}, 1 \mathrm{H}), 7.60(\mathrm{~s}, 1 \mathrm{H}), 7.40-7.20(\mathrm{~m}, 5 \mathrm{H})$, $7.10(\mathrm{~d}, J=8.2 \mathrm{~Hz}, 1 \mathrm{H}), 5.11(\mathrm{~s}, 2 \mathrm{H}), 3.88(\mathrm{~s}, 3 \mathrm{H}) ;{ }^{13} \mathrm{C} \mathrm{NMR}\left(100 \mathrm{MHz}, \mathrm{CD}_{3} \mathrm{SOCD}_{3}\right) \delta 166.82$, $155.13,137.38,133.14,130.47,129.22,128.81,127.97,127.56,124.01,122.51,109.13,52.45$, 43.70; ESI-MS m/z, $283.1(\mathrm{M}+\mathrm{H})^{+}$; ESI-HRMS calcd. for $\mathrm{C}_{16} \mathrm{H}_{15} \mathrm{~N}_{2} \mathrm{O}_{3}(\mathrm{M}+\mathrm{H})^{+}$required 283.1091, found 283.1077.

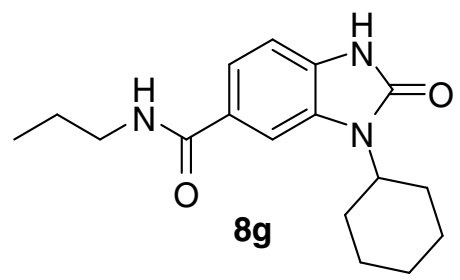

3-Cyclohexyl-2-oxo- $N$-propyl-2,3-dihydro-1H-benzo[d]imidazole-5-carboxamide $8 g:{ }^{1} \mathrm{H}$ $\operatorname{NMR}\left(400 \mathrm{MHz}, \mathrm{CDCl}_{3}\right) \delta 10.74($ br s, $1 \mathrm{H}), 7.77(\mathrm{~s}, 1 \mathrm{H}), 7.37(\mathrm{~d}, J=7.8 \mathrm{~Hz}, 1 \mathrm{H}), 7.12(\mathrm{~d}, J=7.8$ $\mathrm{Hz}, 1 \mathrm{H}), 6.30(\mathrm{~m}, 1 \mathrm{H}), 4.28(\mathrm{~m}, 1 \mathrm{H}), 3.43(\mathrm{dd}, J=6.9,6.7 \mathrm{~Hz}, 2 \mathrm{H}), 2.30-1.20(\mathrm{~m}, 12 \mathrm{H}), 1.00(\mathrm{t}, J=$ $7.3 \mathrm{~Hz}, 3 \mathrm{H}) ;{ }^{13} \mathrm{C} \mathrm{NMR}\left(100 \mathrm{MHz}, \mathrm{CDCl}_{3}\right) \delta 167.73,155.78,130.97,129.82,127.95,119.29$, 109.36, 109.02, 53.17, 42.01, 30.23, 26.02, 25.22, 23.07, 11.58; ESI-MS m/z $302.1(\mathrm{M}+\mathrm{H})^{+}$; ESI-HRMS calcd. for $\mathrm{C}_{17} \mathrm{H}_{24} \mathrm{~N}_{3} \mathrm{O}_{2}(\mathrm{M}+\mathrm{H})^{+}$required 302.1868, found 302.1863. 


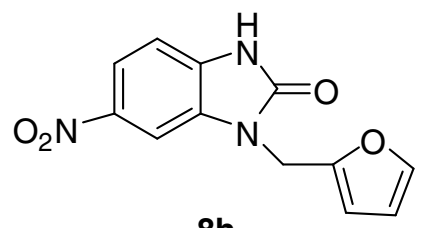

$8 \mathrm{~h}$

1-(Furan-2-ylmethyl)-6-nitro-1H-benzo[d]imidazol-2(3H)-one 8h: ${ }^{1} \mathrm{H}$ NMR (400 MHz, $\left.\left(\mathrm{CD}_{3}\right)_{2} \mathrm{SO}\right) \delta 11.71(\mathrm{br} \mathrm{s}, 1 \mathrm{H}), 8.02(\mathrm{~s}, 1 \mathrm{H}), 7.94(\mathrm{~d}, J=8.3 \mathrm{~Hz}, 1 \mathrm{H}), 7.56(\mathrm{~s}, 1 \mathrm{H}), 7.11(\mathrm{dd}, J=17.4$ $8.3 \mathrm{~Hz}, 1 \mathrm{H}), 6.40(\mathrm{~m}, 2 \mathrm{H}), 5.10(\mathrm{~s}, 2 \mathrm{H}) ;{ }^{13} \mathrm{C} \mathrm{NMR}\left(100 \mathrm{MHz},\left(\mathrm{CD}_{3}\right)_{2} \mathrm{SO}\right) \delta 154.75,149.80,143.71$, 142.00, 134.72, 130.40, 118.87, 111.14, 109.44, 109.08, 104.23, 37.40; ESI-MS m/z $260.2(\mathrm{M}+\mathrm{H})^{+}$; ESI-HRMS calcd. for $\mathrm{C}_{12} \mathrm{H}_{10} \mathrm{~N}_{3} \mathrm{O}_{4}(\mathrm{M}+\mathrm{H})^{+}$required 260.0668, found 260.0666.

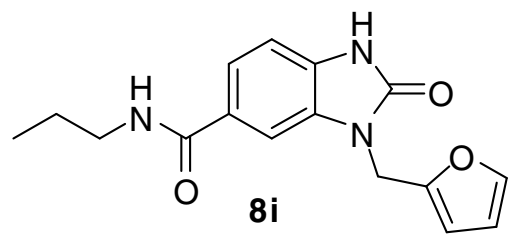

\section{3-(Furan-2-ylmethyl)-2-oxo-N-propyl-2,3-dihydro-1H-benzo[d]imidazole-5-}

carboxamide 8i: ${ }^{1} \mathrm{H}$ NMR $\left(400 \mathrm{MHz}, \mathrm{CDCl}_{3}\right) \delta 11.16$ (br s, 1H), 8.27 (s, 1H), $7.63(\mathrm{~s}, 1 \mathrm{H}), 7.54(\mathrm{~d}$, $J=8.2 \mathrm{~Hz}, 2 \mathrm{H}), 6.97(\mathrm{~d}, J=7.8 \mathrm{~Hz}, 1 \mathrm{H}), 6.37(\mathrm{~d}, J=8.2 \mathrm{~Hz}, 2 \mathrm{H}), 4.99(\mathrm{~s}, 2 \mathrm{H}), 3.16(\mathrm{dd}, J=6.4$, $6.0 \mathrm{~Hz}, 2 \mathrm{H}), 1.49(\mathrm{dd}, J=7.3,6.9 \mathrm{~Hz}, 2 \mathrm{H}), 0.84(\mathrm{t}, J=7.3 \mathrm{~Hz},, 3 \mathrm{H}) ;{ }^{13} \mathrm{C} \mathrm{NMR}\left(100 \mathrm{MHz}, \mathrm{CDCl}_{3}\right)$ $\delta: 166.5,154.7,150.3,143.4,131.1,127.9,121.3,111.1,108.9,108.5,107.8,41.8,37.1,23.0,12.0$; ESI-MS $m / z 300.1(\mathrm{M}+\mathrm{H})^{+}$; ESI-HRMS calcd. for $\mathrm{C}_{16} \mathrm{H}_{18} \mathrm{~N}_{3} \mathrm{O}_{3}(\mathrm{M}+\mathrm{H})^{+}$required 300.3345, found 300.3357.<smiles>CC(C)n1c(=O)[nH]c2ccc(COS(C)(=O)=O)cc21</smiles>

6-((tert-Butyldimethylsilyloxy)methyl)-1-isopropyl-1H-benzo[d]imidazol-2(3H)-one $8 \mathrm{j}$ :

${ }^{1} \mathrm{H}$ NMR $\left(400 \mathrm{MHz}, \mathrm{CDCl}_{3}\right) \delta 10.40($ br s, $1 \mathrm{H}), 7.20(\mathrm{~s}, 1 \mathrm{H}), 7.07(\mathrm{~d}, J=8.2 \mathrm{~Hz}, 1 \mathrm{H}), 6.97(\mathrm{~d}, J=$ $8.2 \mathrm{~Hz}, 1 \mathrm{H}), 4.77(\mathrm{~s}, 3 \mathrm{H}), 1.56(\mathrm{~s}, 3 \mathrm{H}), 1.55(\mathrm{~s}, 3 \mathrm{H}), 0.95(\mathrm{~s}, 9 \mathrm{H}), 0.11(\mathrm{~s}, 6 \mathrm{H}),{ }^{13} \mathrm{C} \mathrm{NMR}(100 \mathrm{MHz}$ 
$\left.\mathrm{CDCl}_{3}\right) \delta: 155.58,134.54,129.18,127.36$ 119.13, 109.43, 107.68, 65.32, 44.64, 26.05, 20.39, 18.50, -.05; ESI-MS $m / z, 321.2(\mathrm{M}+\mathrm{H})^{+}$; ESI-HRMS calcd. for $\mathrm{C}_{17} \mathrm{H}_{29} \mathrm{~N}_{2} \mathrm{O}_{2} \mathrm{Si}(\mathrm{M}+\mathrm{H})^{+}$required 321.2006, found 321.1993.

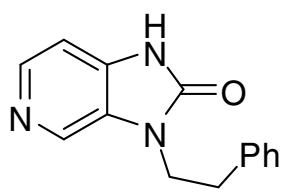

8k

3-Phenethyl-1H-imidazo[4,5-c]pyridin-2(3H)-one 8k: ${ }^{1} \mathrm{H} \mathrm{NMR}\left(400 \mathrm{MHz},\left(\mathrm{CD}_{3}\right)_{2} \mathrm{SO}\right) \delta$ $11.26(\mathrm{br} \mathrm{s}, 1 \mathrm{H}), 8.20(\mathrm{~s}, 1 \mathrm{H}), 8.07(\mathrm{~d}, J=5.0 \mathrm{~Hz}, 1 \mathrm{H}), 7.18(\mathrm{~m}, 5 \mathrm{H}), 6.95(\mathrm{~d}, J=5.0 \mathrm{~Hz}, 1 \mathrm{H}), 4.01$ (t, $J=7.3 \mathrm{~Hz}, 2 \mathrm{H}), 2.93(\mathrm{t}, J=7.3 \mathrm{~Hz}, 2 \mathrm{H}) ;{ }^{13} \mathrm{C}$ NMR $\left(100 \mathrm{MHz},\left(\mathrm{CD}_{3}\right)_{2} \mathrm{SO}\right) \delta$ 154.01, 145.1, 142.67, 138.78, 134.77, 129.36, 128.87, 128.23, 126.94, 104.78, 42.20, 34.20; ESI-MS m/z 240.1 $(\mathrm{M}+\mathrm{H})^{+}$; ESI-HRMS calcd. for $\mathrm{C}_{14} \mathrm{H}_{14} \mathrm{~N}_{3} \mathrm{O}(\mathrm{M}+\mathrm{H})^{+}$required 240.1144, found 240.1131 .

General procedure for synthesis of benzimidazol-2-ones from aryl iodides: A Schlenk tube was charged with aryl iodide $(0.5 \mathrm{mmol}), \mathrm{CuI}(10 \mathrm{mg}, 0.05 \mathrm{mmol}), L$-Proline $(12 \mathrm{mg}, 0.1$ mmol), and $\mathrm{K}_{2} \mathrm{CO}_{3}(138 \mathrm{mg}, 1.0 \mathrm{mmol})$, evacuated and backfilled with argon. Amine (0.5 mmol) and DMSO $(1 \mathrm{~mL})$ were successively added. The reaction mixture was stirred at $50{ }^{\circ} \mathrm{C}$ until the coupling was completed detected by TLC. The solution was then heated at $130{ }^{\circ} \mathrm{C}$ until the coupling product was consumed monitored by TLC. The cold mixture was partitioned between ethyl acetate and saturated $\mathrm{NH}_{4} \mathrm{Cl}$. The organic layer was washed with brine, dried over $\mathrm{Na}_{2} \mathrm{SO}_{4}$ and concentrated in vacuo. The residue was purified by column chromatography on silica gel to provide the desired product.

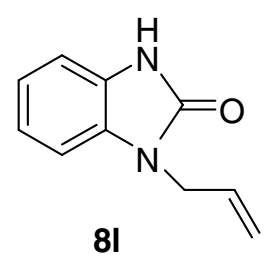


1-Allyl-1H-benzo[d]imidazol-2(3H)-one 8l: ${ }^{1} \mathrm{H}$ NMR $\left(400 \mathrm{MHz}, \mathrm{CDCl}_{3}\right) \delta 10.42(\mathrm{~s}, 1 \mathrm{H})$, 7.30-7.01 (m, 4H), $5.93(\mathrm{~m}, 1 \mathrm{H}), 5.25(\mathrm{~m}, 2 \mathrm{H}), 4.54(\mathrm{~d}, J=5.0 \mathrm{~Hz}, 2 \mathrm{H}) ;{ }^{13} \mathrm{C} \mathrm{NMR}(100 \mathrm{MHz}$, $\left.\mathrm{CDCl}_{3}\right) \delta 155.9,131.9,130.1,128.3,121.7,121.2,117.5,109.9,108.4,43.1 ;$ ESI-MS $\mathrm{m} / \mathrm{z} 175.1$ $(\mathrm{M}+\mathrm{H})^{+}$; ESI-HRMS calcd. for $\mathrm{C}_{10} \mathrm{H}_{10} \mathrm{~N}_{2} \mathrm{O}\left(\mathrm{M}^{+}\right)$required 174.0793, found 174.0794.<smiles>O=c1[nH]c2ccccc2n1C1CCC(c2ccccc2)CC1</smiles>

1-(4-Phenylcyclohexyl)-1H-benzo[d]imidazol-2(3H)-one 8m: ${ }^{1} \mathrm{H} \mathrm{NMR}\left(400 \mathrm{MHz}, \mathrm{CDCl}_{3}\right)$ $\delta 10.28(\mathrm{~s}, 1 \mathrm{H}), 7.48-6.69(\mathrm{~m}, 9 \mathrm{H}), 4.51(\mathrm{~m}, 1 \mathrm{H}), 3.19(\mathrm{~m}, 1 \mathrm{H}), 2.47(\mathrm{~m}, 2 \mathrm{H}), 2.29(\mathrm{~m}, 2 \mathrm{H}), 2.01(\mathrm{~m}$, 2H), $1.74(\mathrm{~m}, 2 \mathrm{H}) ;{ }^{13} \mathrm{C}$ NMR $\left(100 \mathrm{MHz}, \mathrm{CDCl}_{3}\right) \delta 155.4,143.3,129.1,128.6,128.2,127.6,125.8$, 121.0, 120.9, 109.7, 109.6, 52.1, 35.1, 32.0, 25.2, 22.8, 14.2; ESI-MS $m / z .293 .2(\mathrm{M}+\mathrm{H})^{+}$; ESI-HRMS calcd. for $\mathrm{C}_{19} \mathrm{H}_{21} \mathrm{~N}_{2} \mathrm{O}(\mathrm{M}+\mathrm{H})^{+}$required 293.1576, found 293.1577.<smiles>CC(C)(C)OC(=O)N1CCC(n2c(=O)[nH]c3ccccc32)CC1</smiles>

tert-Butyl 4-(2-oxo-2,3-dihydrobenzo[d]imidazol-1-yl)piperidine-1-carboxylate $8 \mathrm{n}:{ }^{1} \mathrm{H}$ NMR (400 MHz, $\left.\mathrm{CDCl}_{3}\right) \delta 10.60(\mathrm{~s}, 1 \mathrm{H}), 7.29-7.15(\mathrm{~m}, 4 \mathrm{H}), 4.50(\mathrm{~m}, 1 \mathrm{H}), 4.32(\mathrm{~m}, 2 \mathrm{H}), 2.89(\mathrm{~m}$, 2H), $2.36(\mathrm{~m}, 2 \mathrm{H}), 1.85(\mathrm{~m}, 2 \mathrm{H}), 1.51(\mathrm{~s}, 9 \mathrm{H}) ;{ }^{13} \mathrm{C} \mathrm{NMR}\left(100 \mathrm{MHz}, \mathrm{CDCl}_{3}\right) \delta 155.4,154.8,128.9$, 128.3, 121.4, 121.1, 110.1, 109.4, 80.5, 50.8, 29.2, 28.5; ESI-MS $m / z$ 318.2 $(\mathrm{M}+\mathrm{H})^{+}$; ESI-HRMS calcd. for $\mathrm{C}_{17} \mathrm{H}_{24} \mathrm{~N}_{3} \mathrm{O}_{3}(\mathrm{M}+\mathrm{H})^{+}$required 318.1739, found 318.1741.<smiles>CC(=O)c1ccc2[nH]c(=O)n(Cc3ccco3)c2c1</smiles> 
6-Acetyl-1-(furan-2-ylmethyl)-1H-benzo[d]imidazol-2(3H)-one 8o: ${ }^{1} \mathrm{H}$ NMR (400 MHz, $\left.\left(\mathrm{CD}_{3}\right)_{2} \mathrm{SO}\right) \delta 11.33(\mathrm{~s}, 1 \mathrm{H}), 7.68(\mathrm{~d}, J=7.8 \mathrm{~Hz}, 2 \mathrm{H}), 7.54(\mathrm{~s}, 1 \mathrm{H}), 7.03(\mathrm{~d}, J=7.8 \mathrm{~Hz}, 1 \mathrm{H}), 6.42(\mathrm{~s}$, $1 \mathrm{H}), 6.36(\mathrm{~s}, 1 \mathrm{H}), 5.05(\mathrm{~s}, 2 \mathrm{H}), 2.49(\mathrm{~s}, 3 \mathrm{H}) ;{ }^{13} \mathrm{C} \mathrm{NMR}\left(100 \mathrm{MHz},\left(\mathrm{CD}_{3}\right)_{2} \mathrm{SO}\right) \delta$ 197.1, 154.6, 150.2, $143.5,132.9,130.7,130.4,123.8,111.1,109.1,108.8,108.1,37.2,27.6$; ESI-MS m/z 255.1 (M $\mathrm{H})^{+}$; ESI-HRMS calcd. for $\mathrm{C}_{14} \mathrm{H}_{13} \mathrm{~N}_{2} \mathrm{O}_{3}(\mathrm{M}+\mathrm{H})^{+}$required 257.0921, found 257.0920.

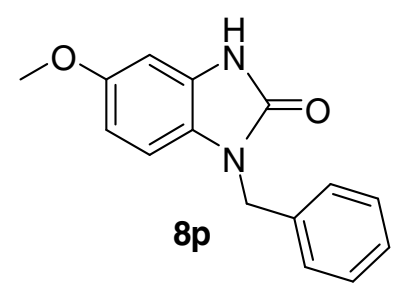

1-Benzyl-5-methoxy-1H-benzo[d]imidazol-2(3H)-one 8p: ${ }^{1} \mathrm{H}$ NMR $\left(400 \mathrm{MHz}, \mathrm{CDCl}_{3}\right) \delta$ $10.71(\mathrm{~s}, 1 \mathrm{H}), 7.31(\mathrm{~m}, 5 \mathrm{H}), 6.76-6.55(\mathrm{~m}, 3 \mathrm{H}), 5.08(\mathrm{~s}, 2 \mathrm{H}), 3.75(\mathrm{~s}, 3 \mathrm{H}) ;{ }^{13} \mathrm{C} \mathrm{NMR}(100 \mathrm{MHz}$, $\left.\mathrm{CDCl}_{3}\right) \delta 155.82,136.33,129.01,128.88,127.76,127.32,124.21,108.99,107.63,96.54,56.01$, 44.62; ESI-MS m/z $255.2(\mathrm{M}+\mathrm{H})^{+}$; ESI-HRMS calcd. for $\mathrm{C}_{15} \mathrm{H}_{14} \mathrm{~N}_{2} \mathrm{O}_{2}\left(\mathrm{M}^{+}\right)$required 254.1055, found 254.1046.<smiles>C=CCn1c(=O)[nH]c2ccc(Br)cc21</smiles>

1-Allyl-6-bromo-1H-benzo[d]imidazol-2(3H)-one 8q: ${ }^{1} \mathrm{H} \mathrm{NMR}\left(400 \mathrm{MHz}, \mathrm{CDCl}_{3}\right) \delta 9.96$ (s, 1H), $7.18(\mathrm{~d}, J=8.3 \mathrm{~Hz}, 1 \mathrm{H}), 7.10(\mathrm{~s}, 1 \mathrm{H}), 6.98(\mathrm{~d}, J=8.3 \mathrm{~Hz}, 1 \mathrm{H}), 5.88(\mathrm{~m}, 1 \mathrm{H}), 5.26(\mathrm{~m}, 2 \mathrm{H})$, $4.49(\mathrm{~s}, 2 \mathrm{H}) ;{ }^{13} \mathrm{C} \mathrm{NMR}\left(100 \mathrm{MHz}, \mathrm{CDCl}_{3}\right) \delta 154.1,131.3,127.1,123.2,119.6,118.0,111.7,110.9$, 105.6, 43.3; ESI-MS m/z $253.1(\mathrm{M}+\mathrm{H})^{+}$; ESI-HRMS calcd. for $\mathrm{C}_{10} \mathrm{H}_{9} \mathrm{~N}_{2} \mathrm{OBr}\left(\mathrm{M}^{+},{ }^{79} \mathrm{Br}\right)$ required 251.9898, found 251.9892. 


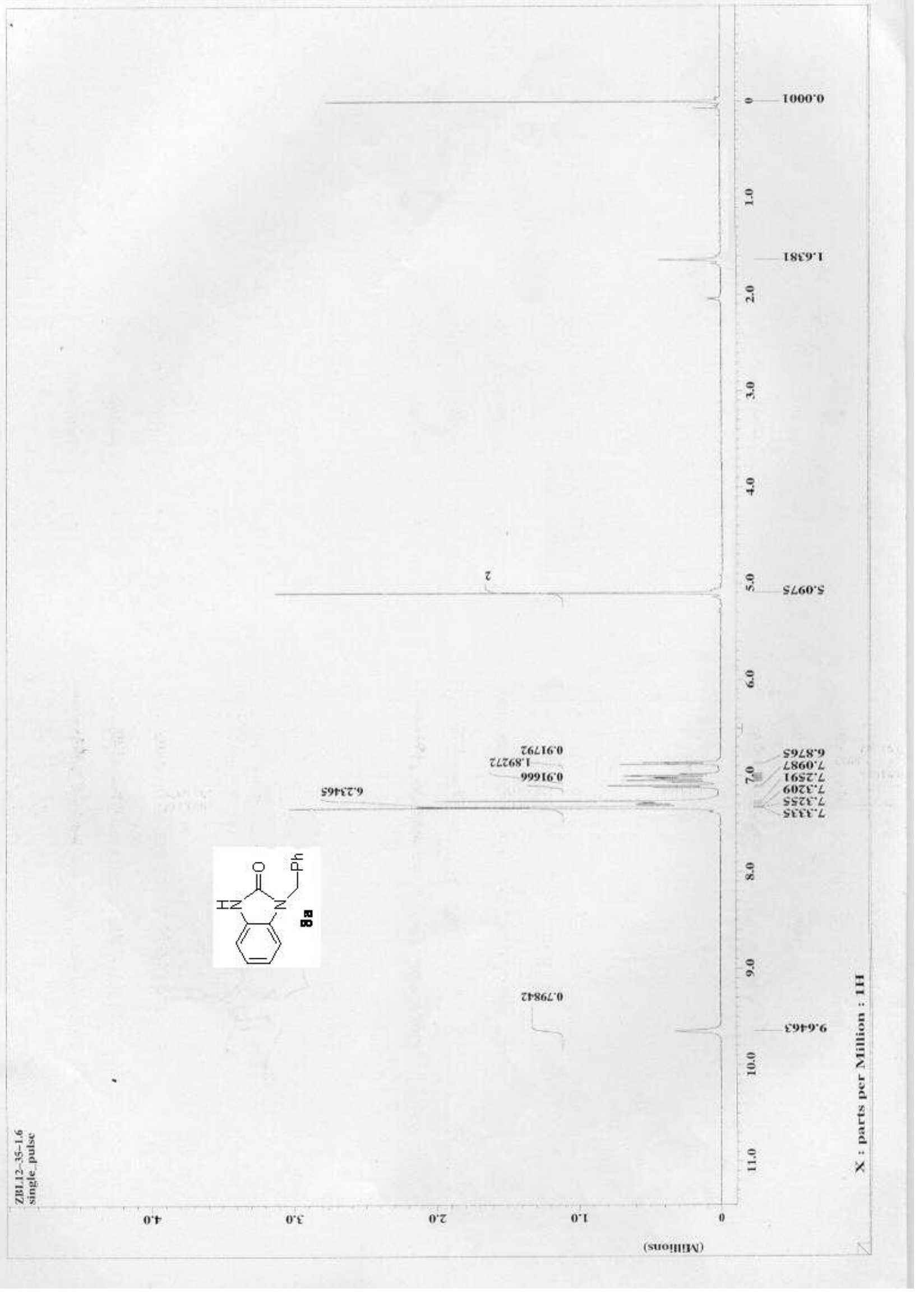




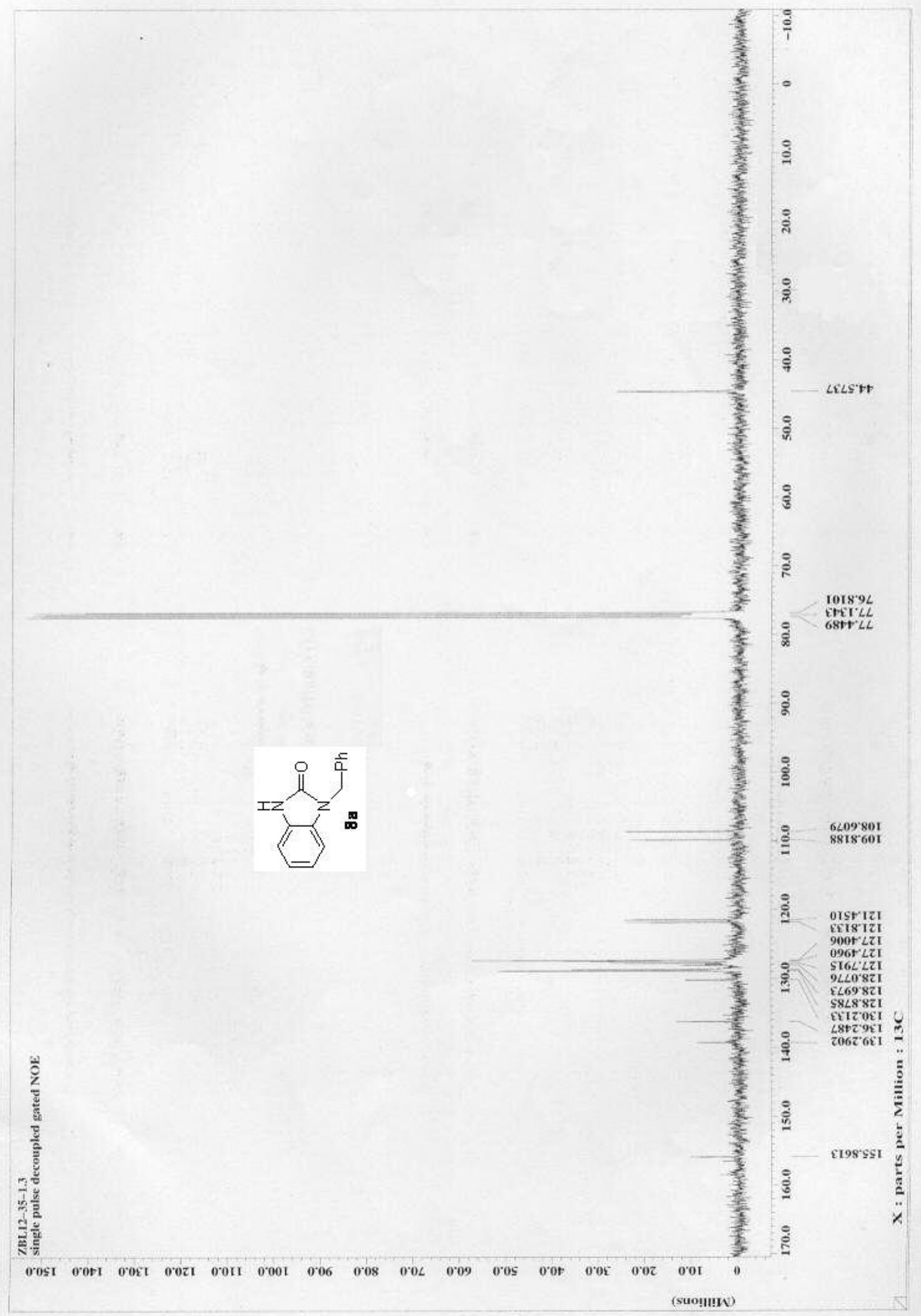




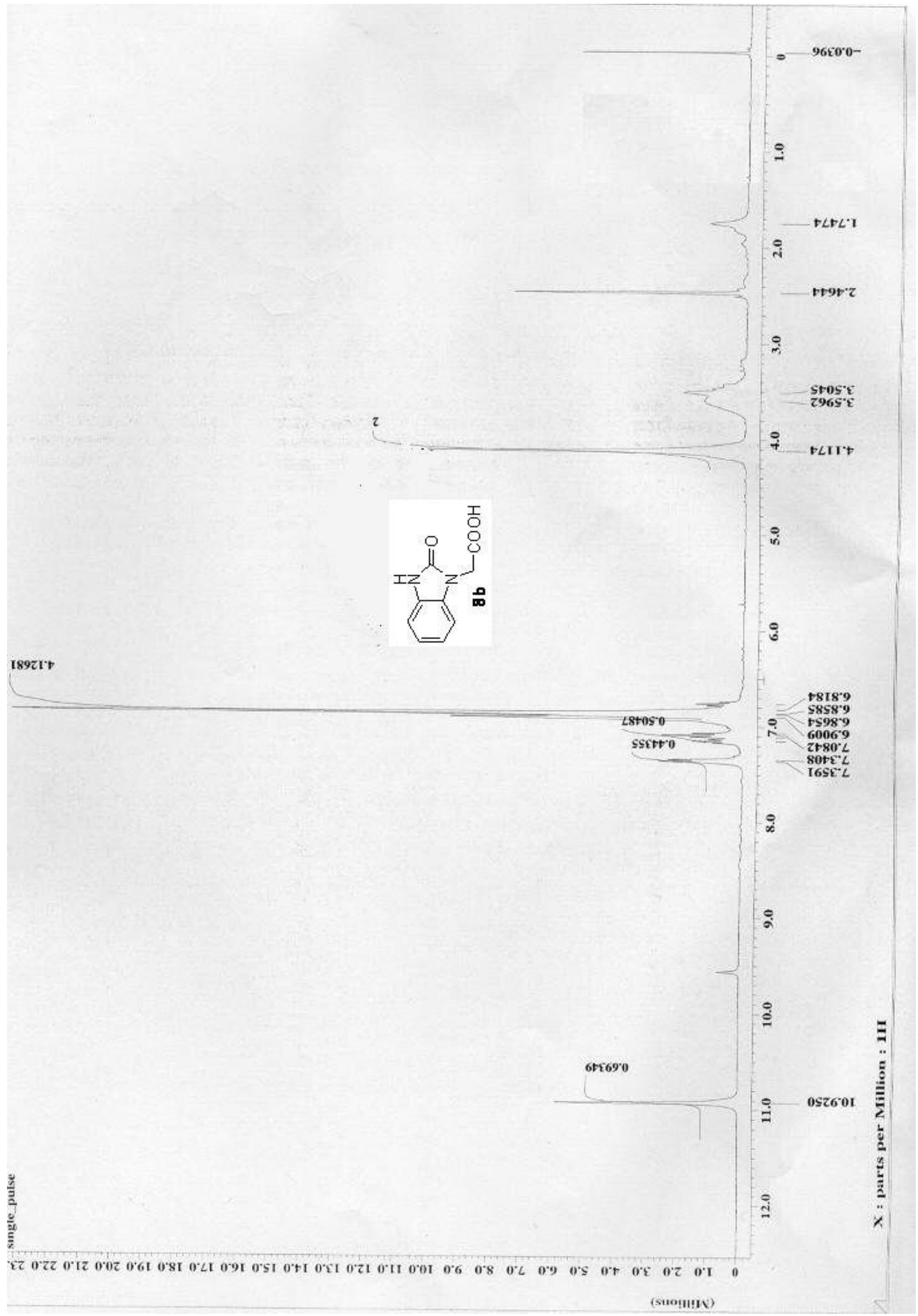




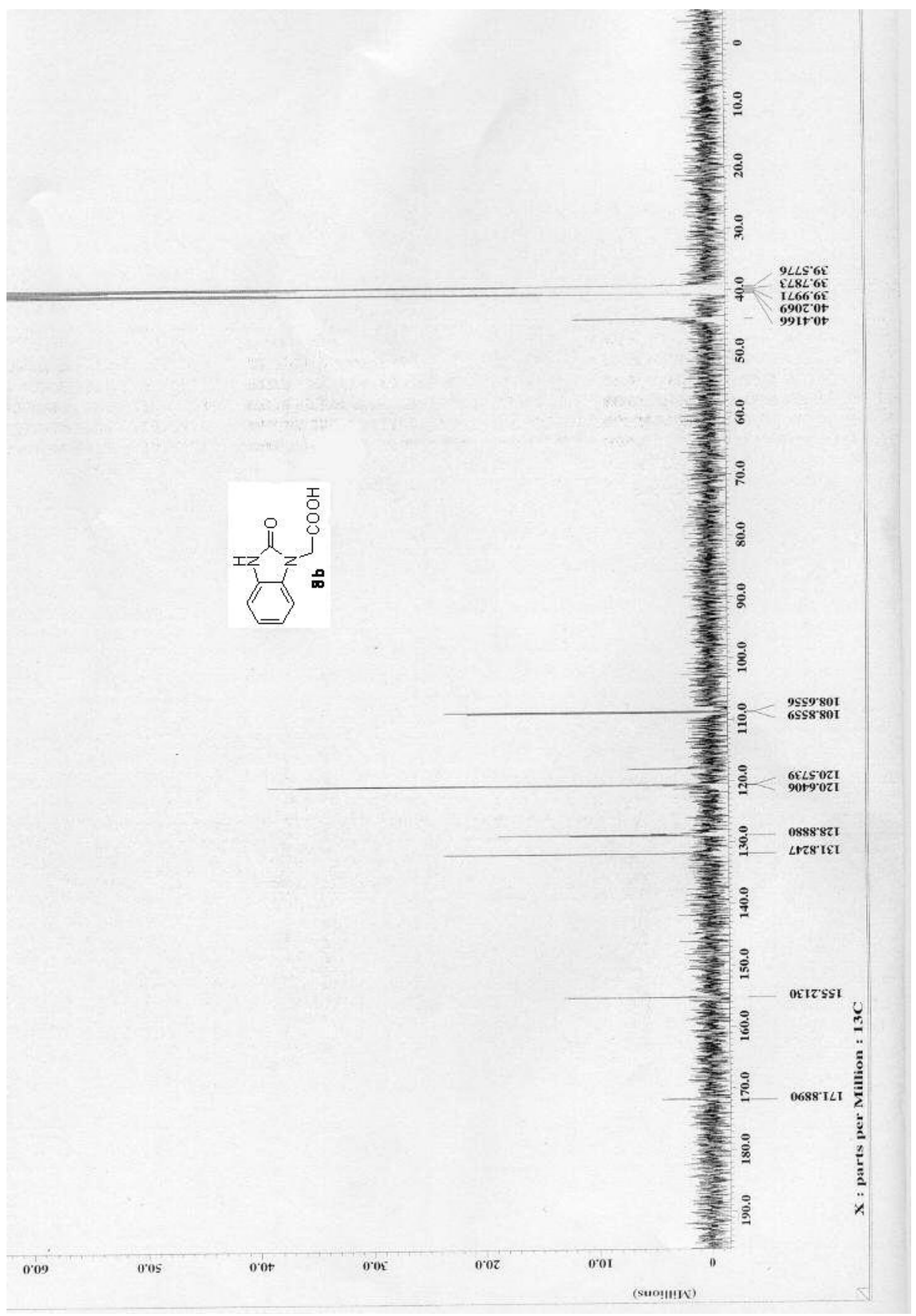




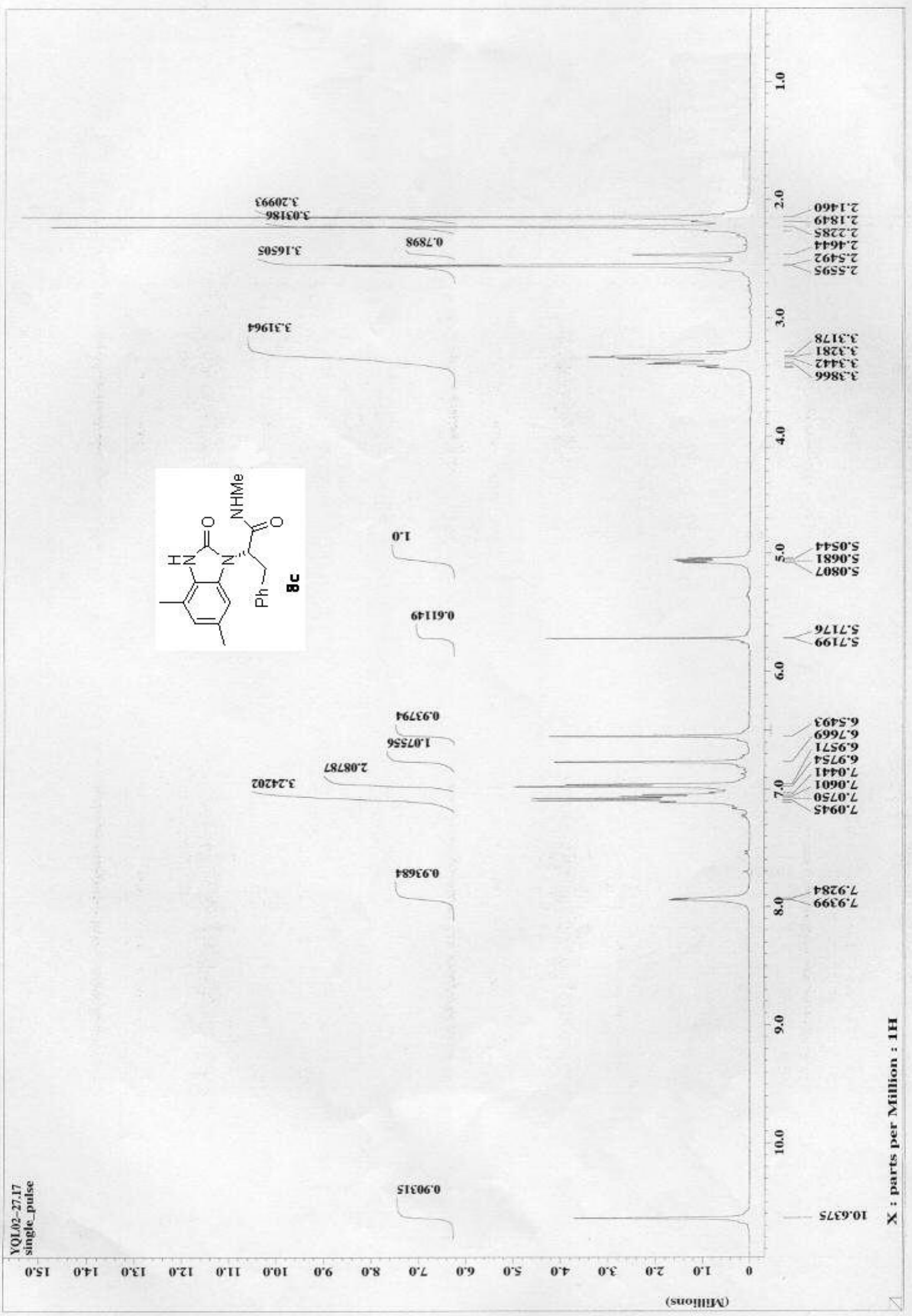




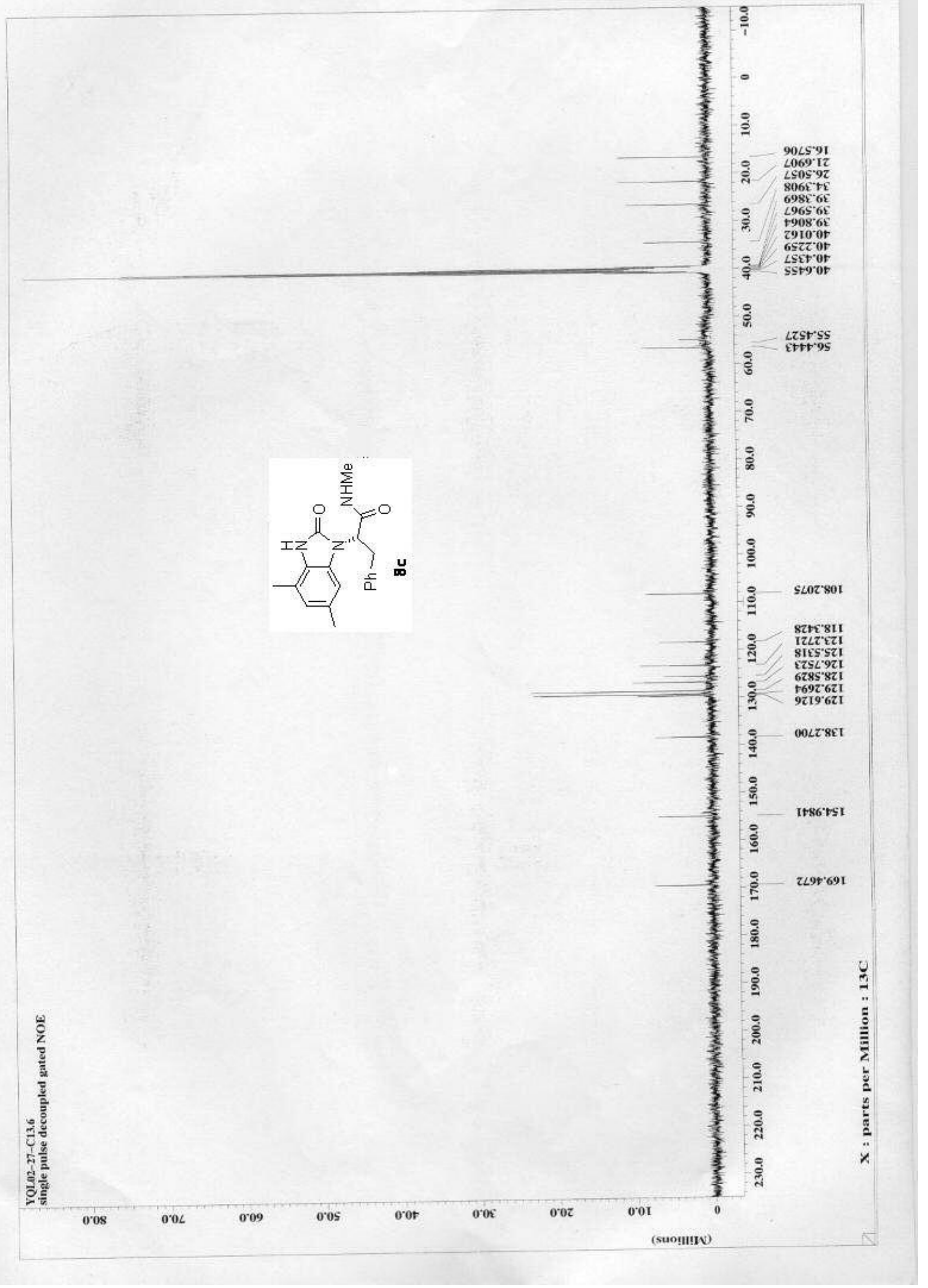




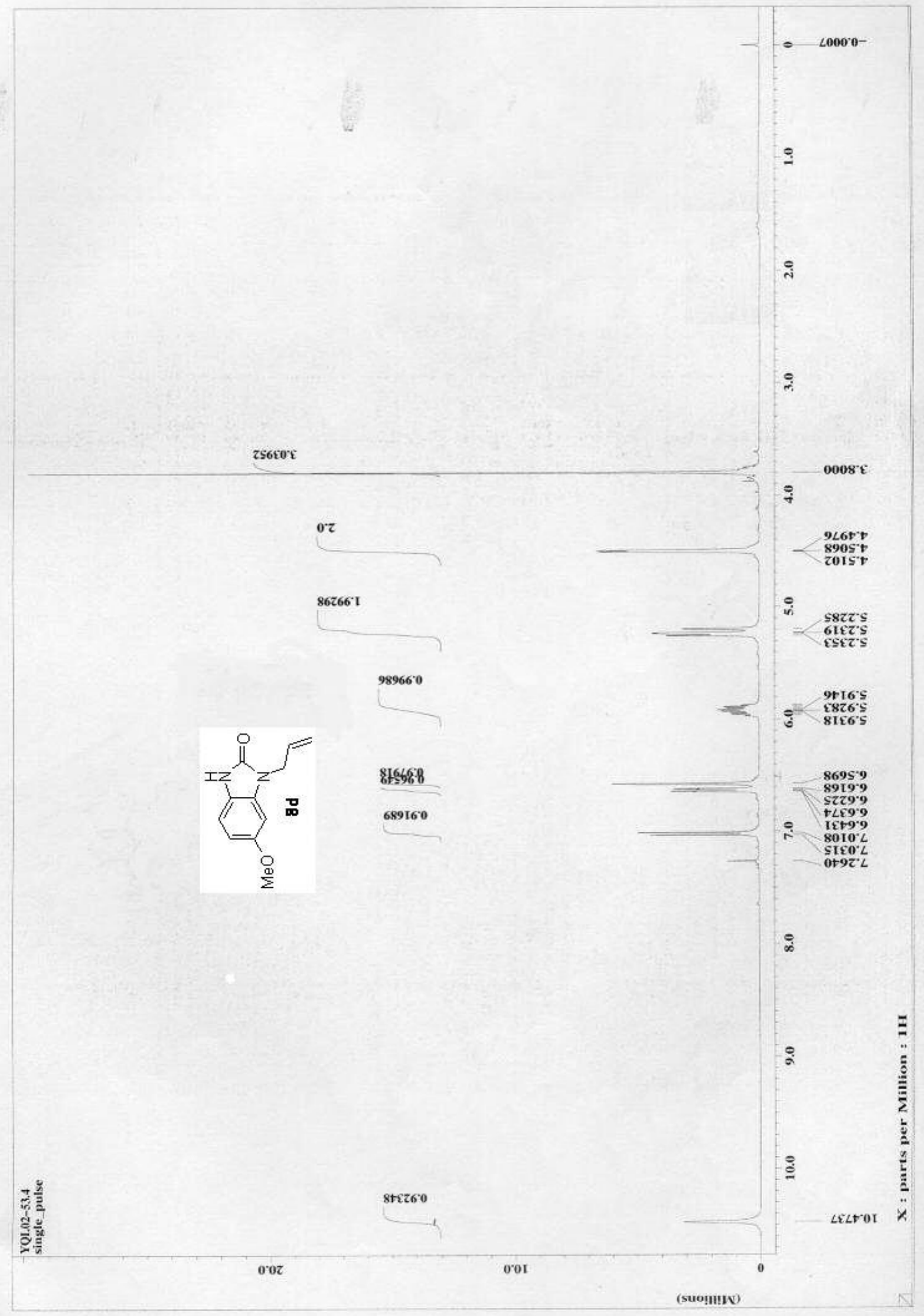




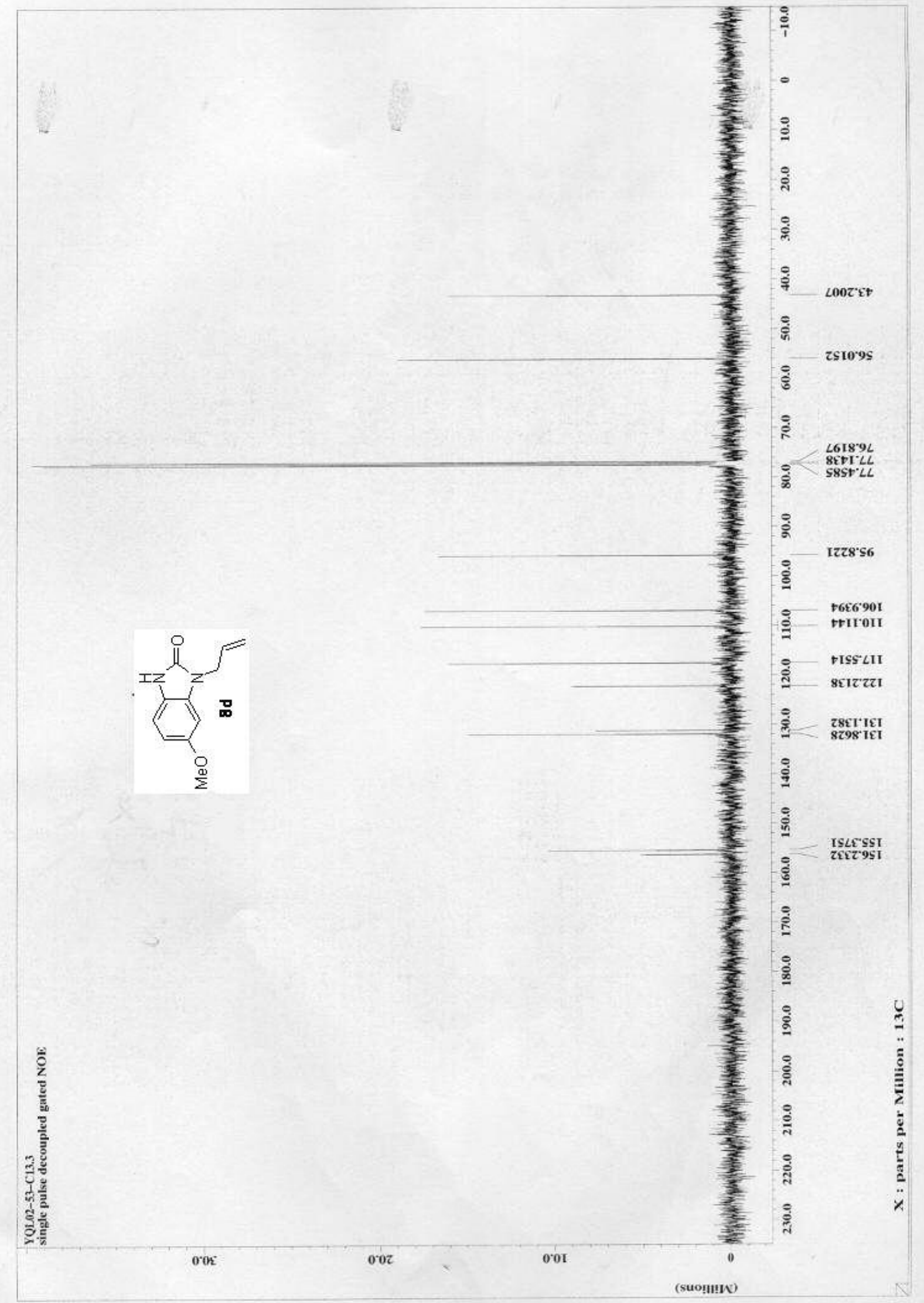




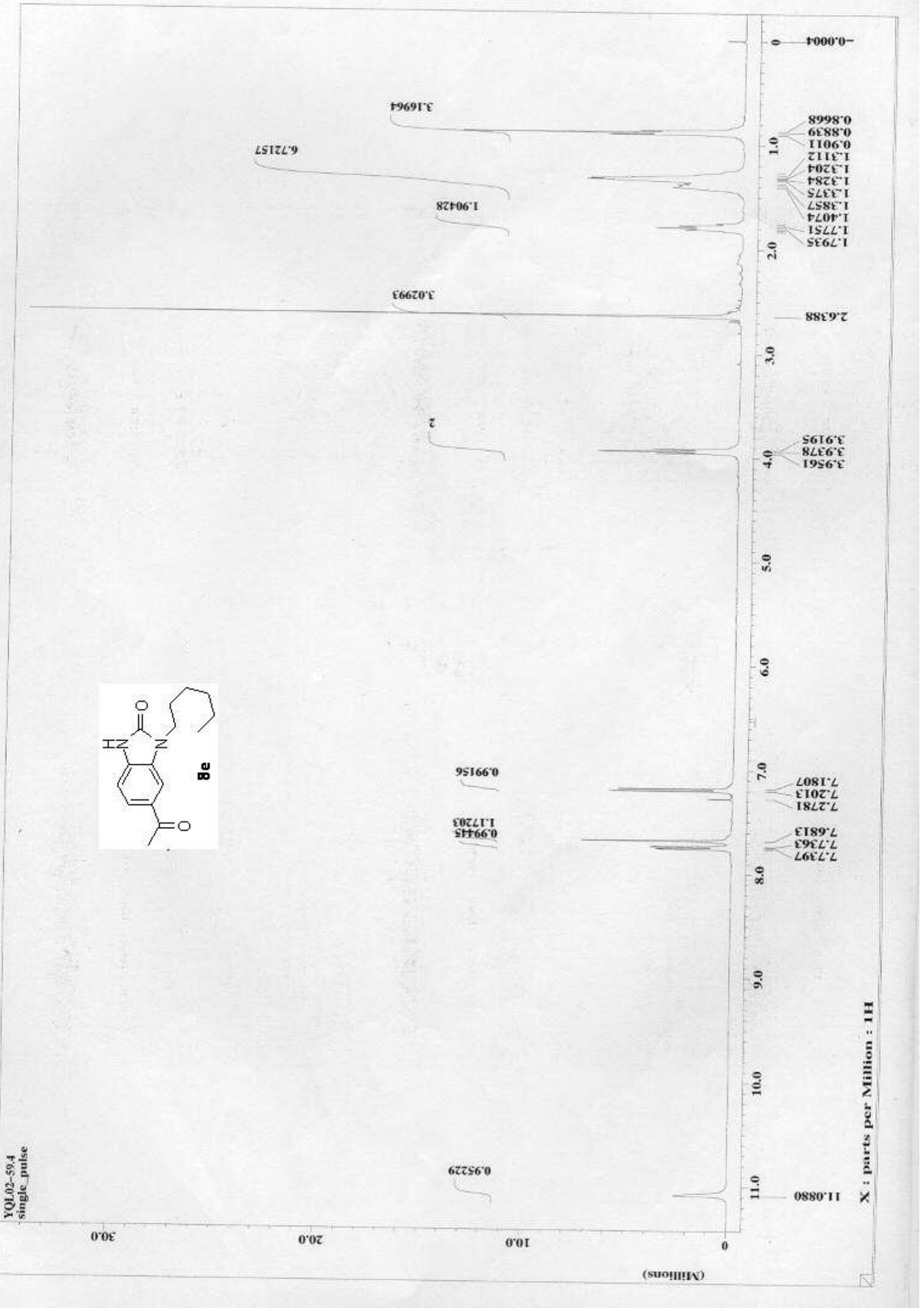




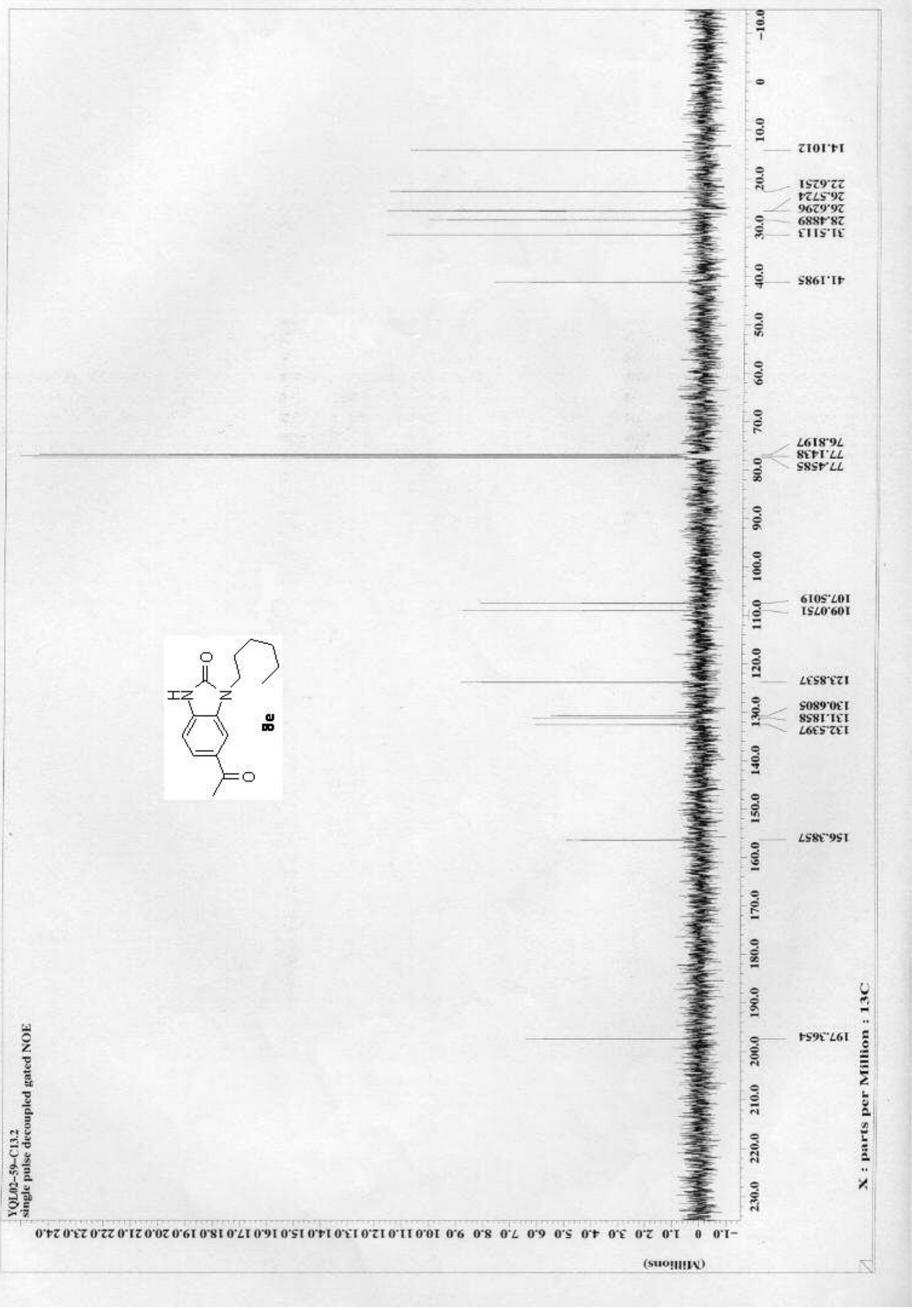




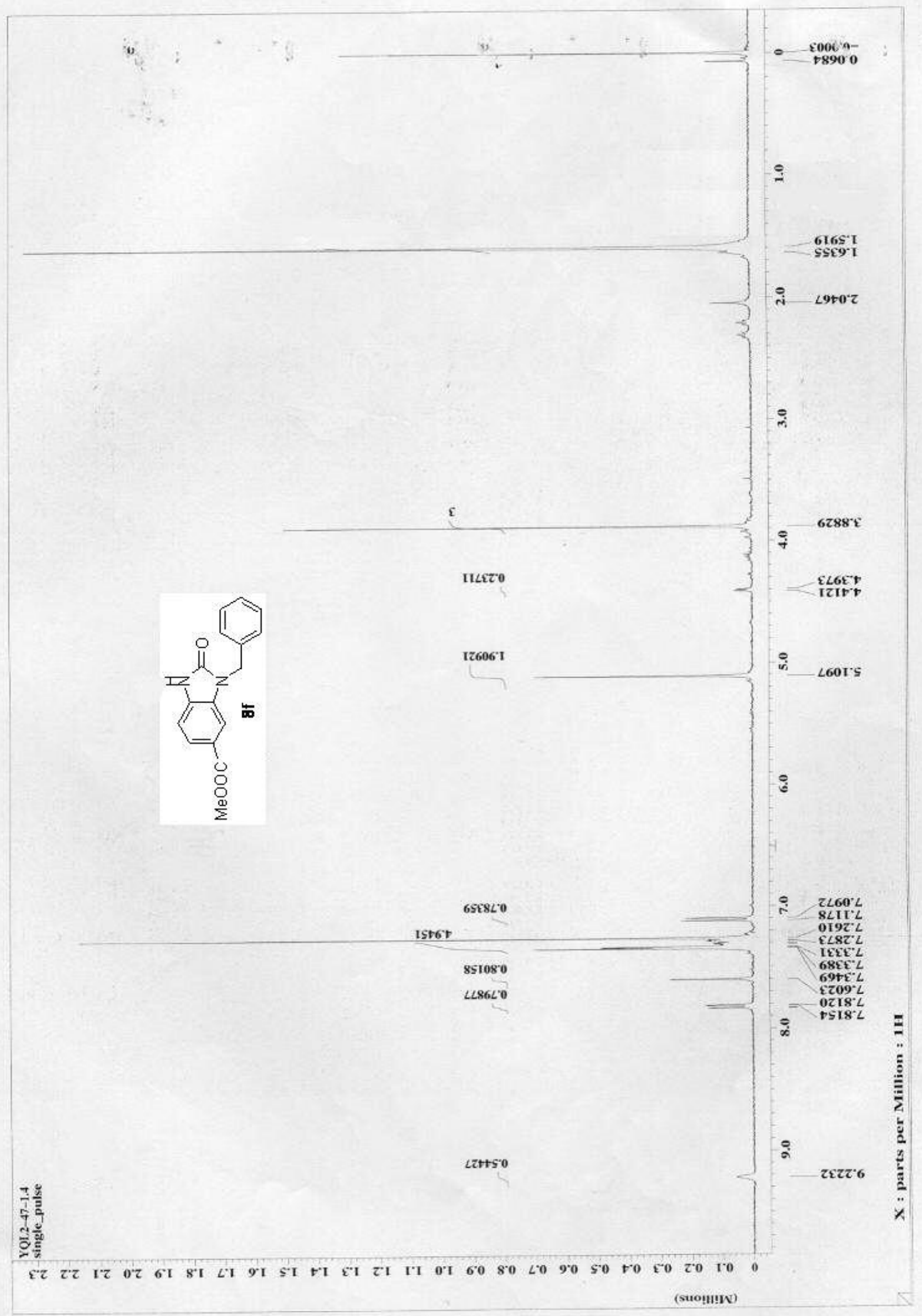




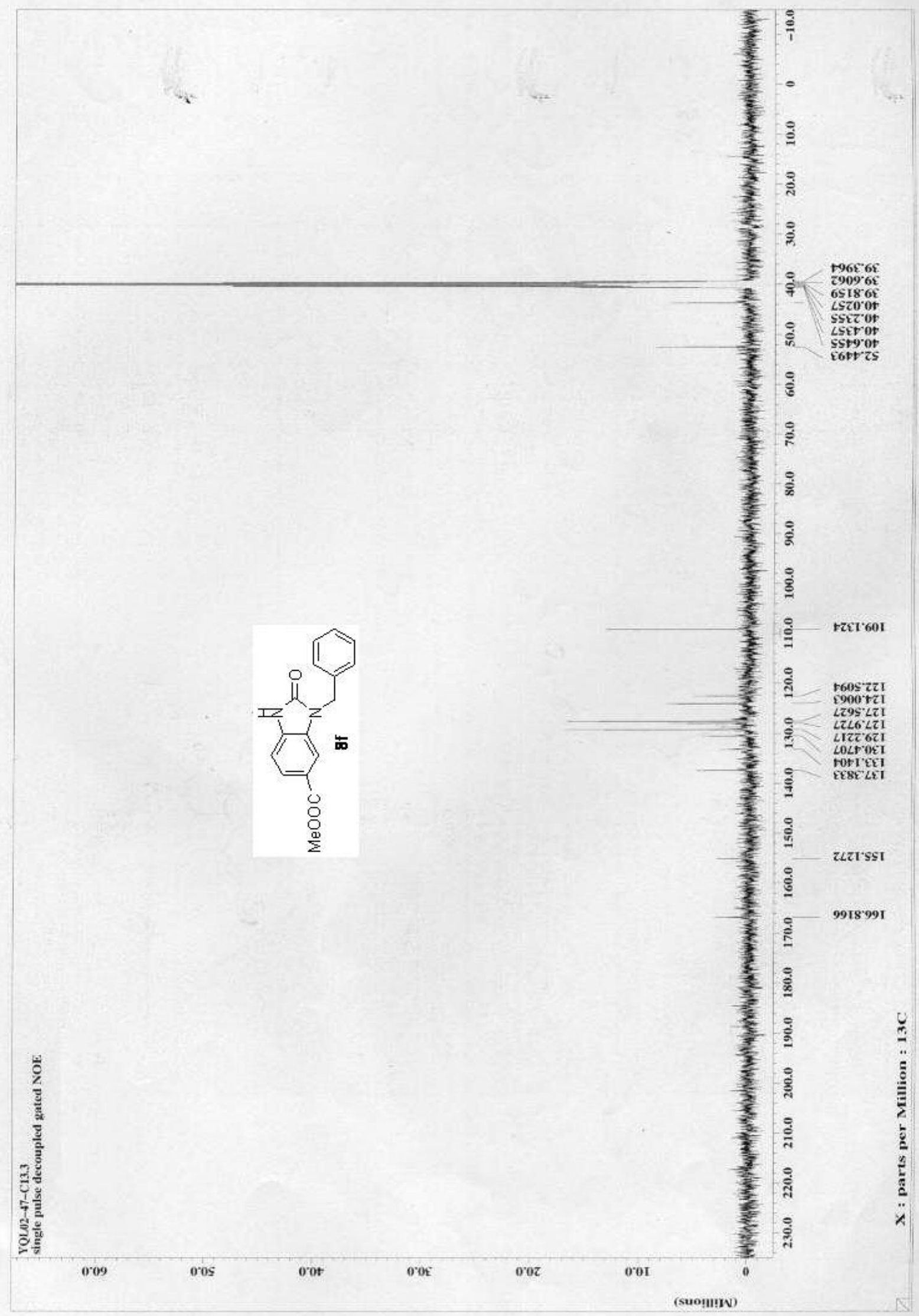




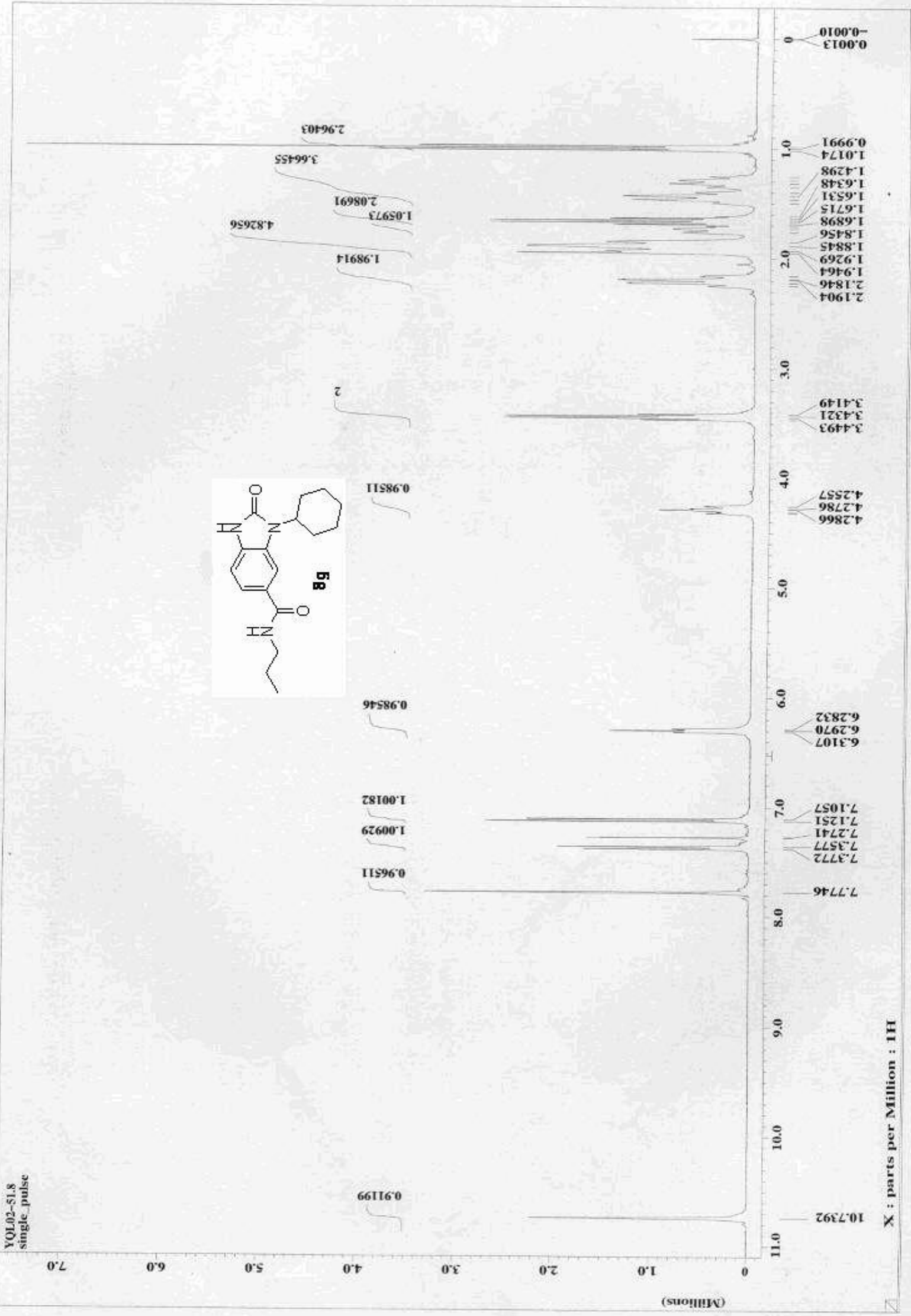




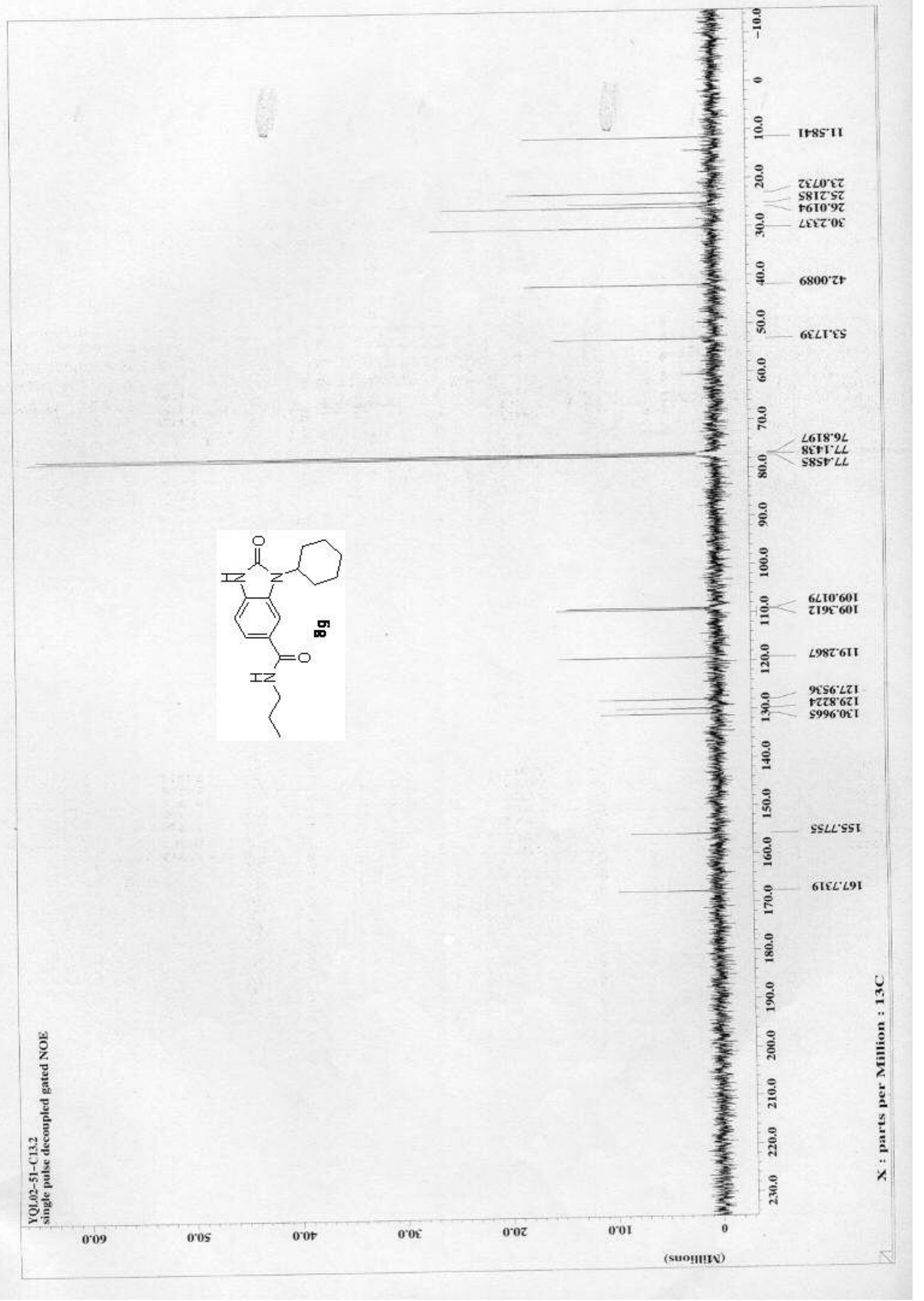




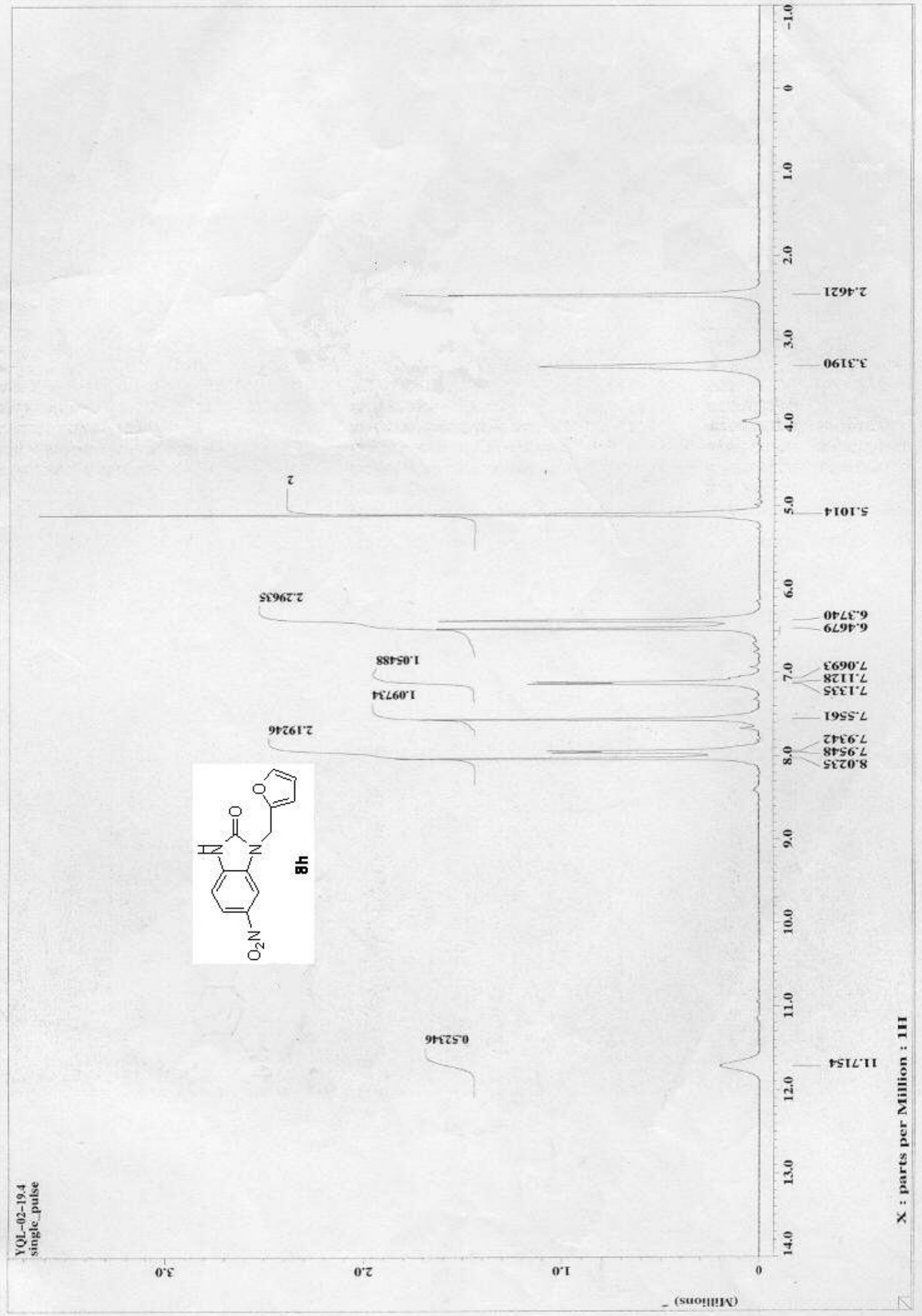




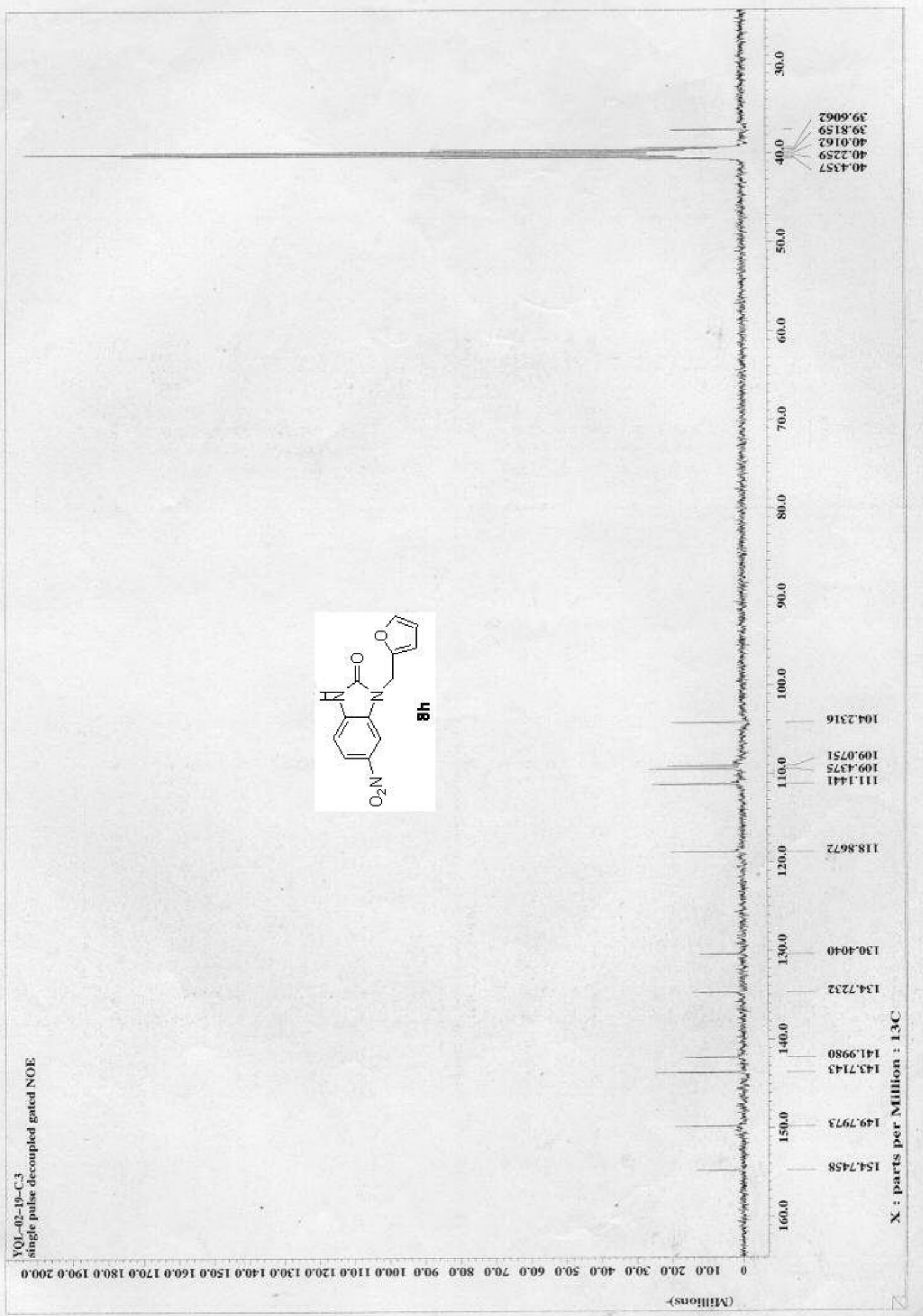




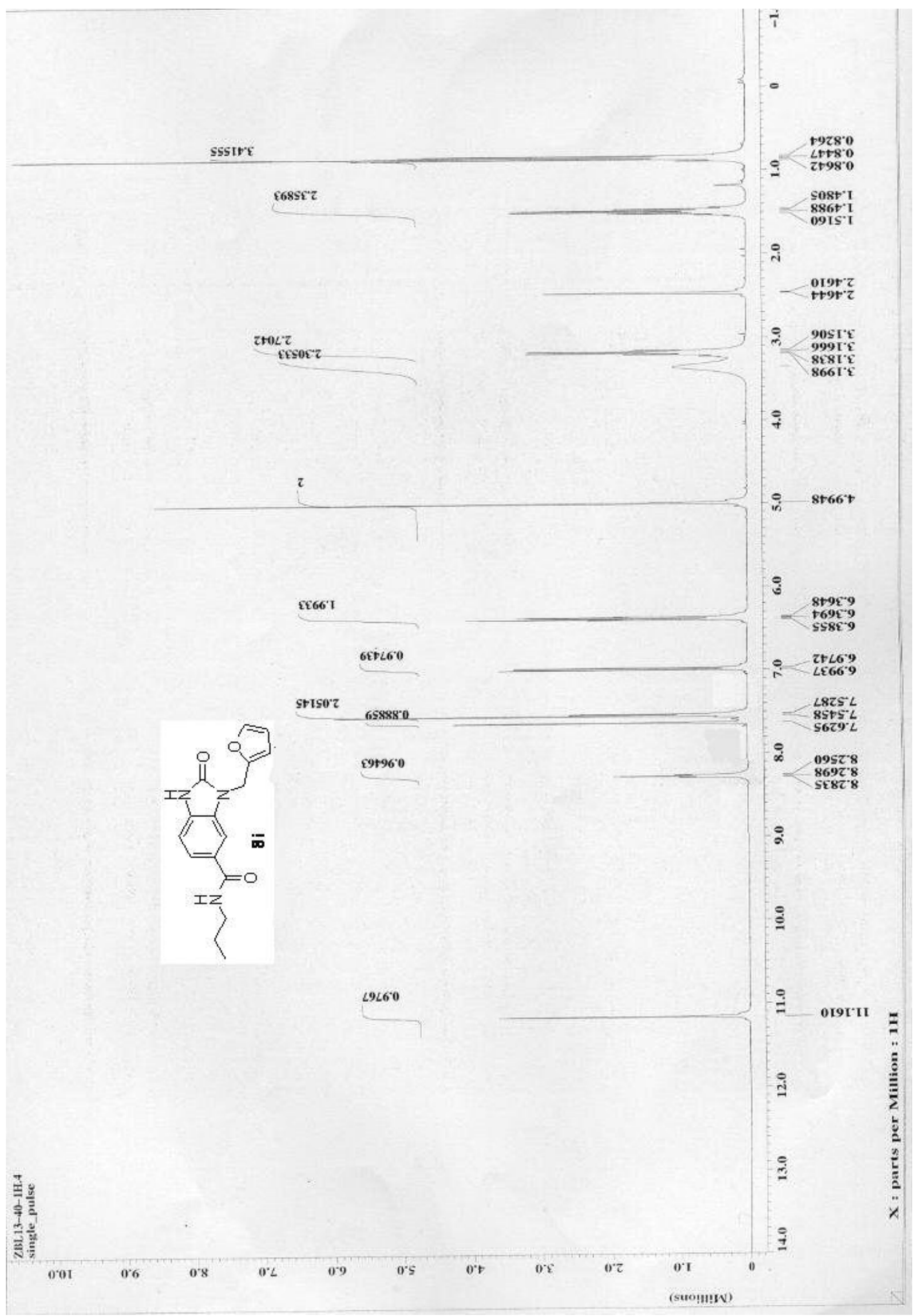




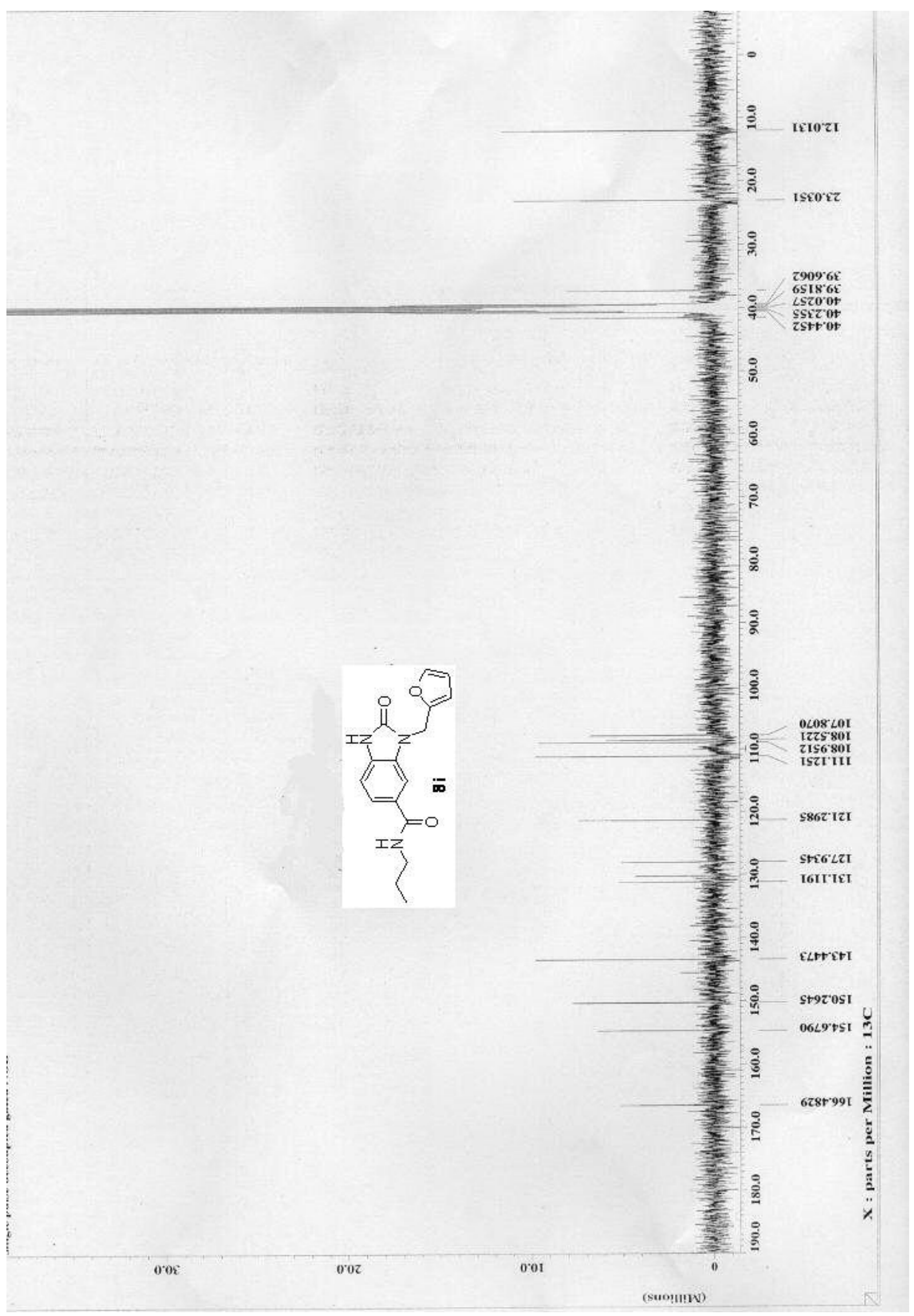




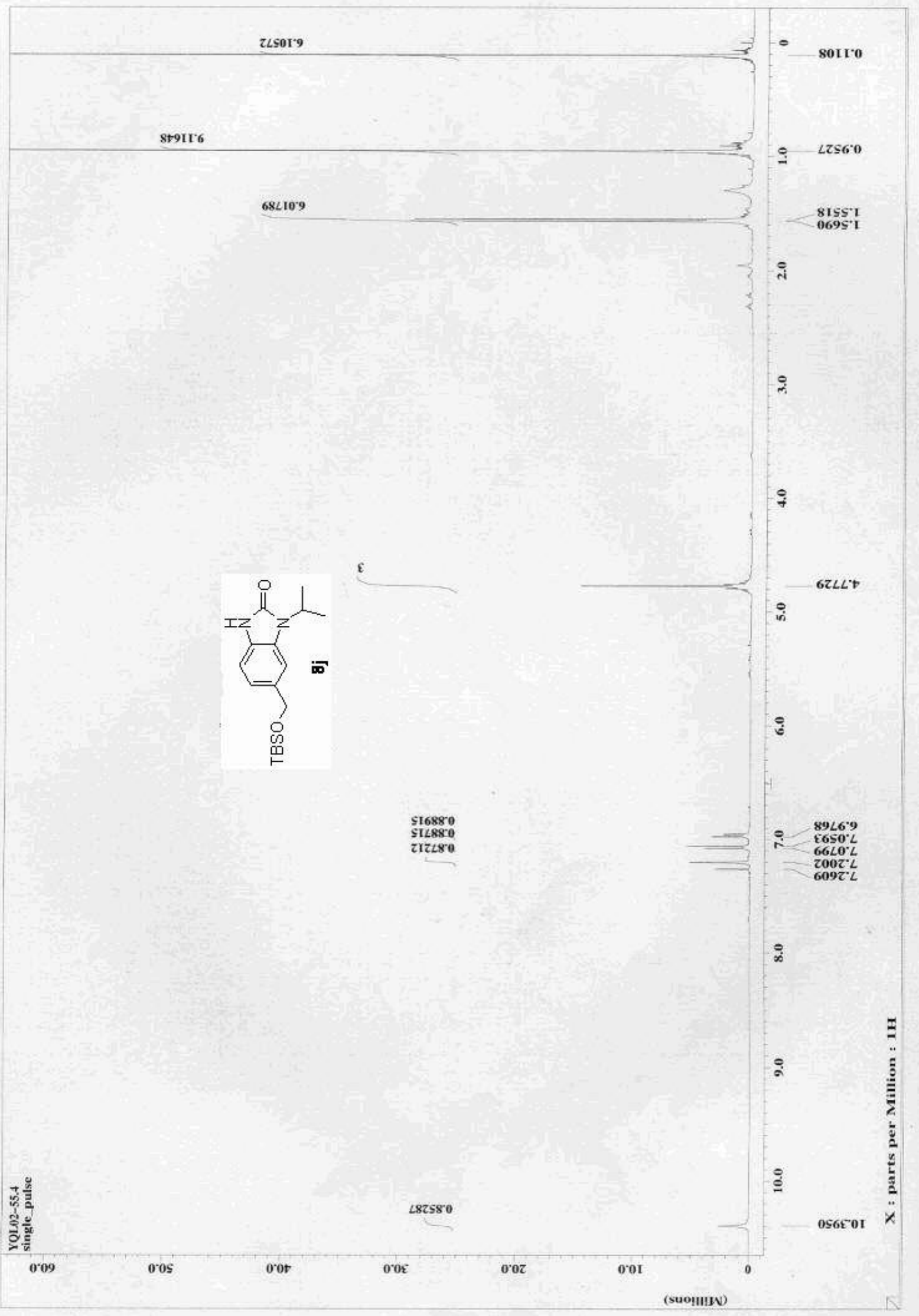




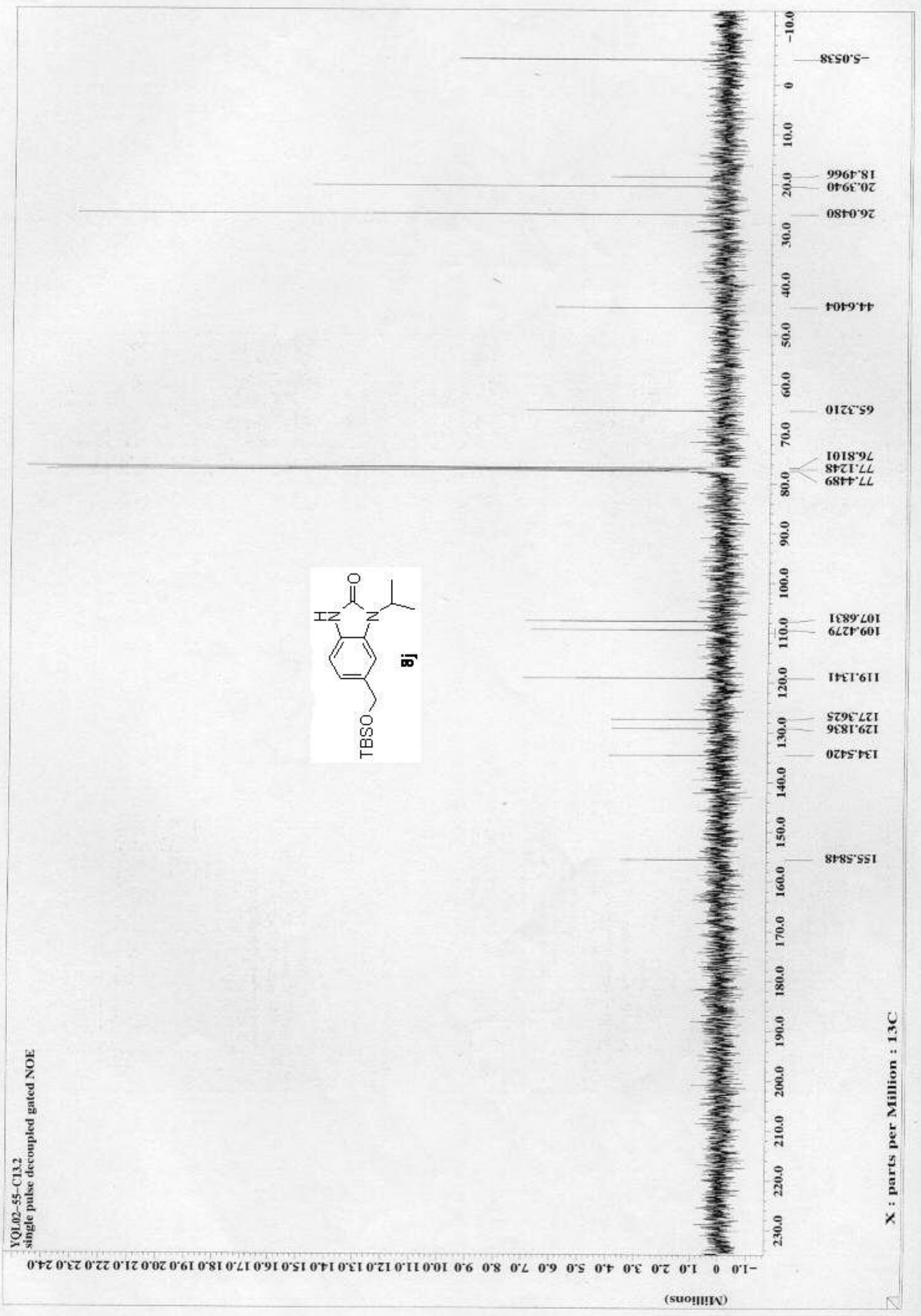




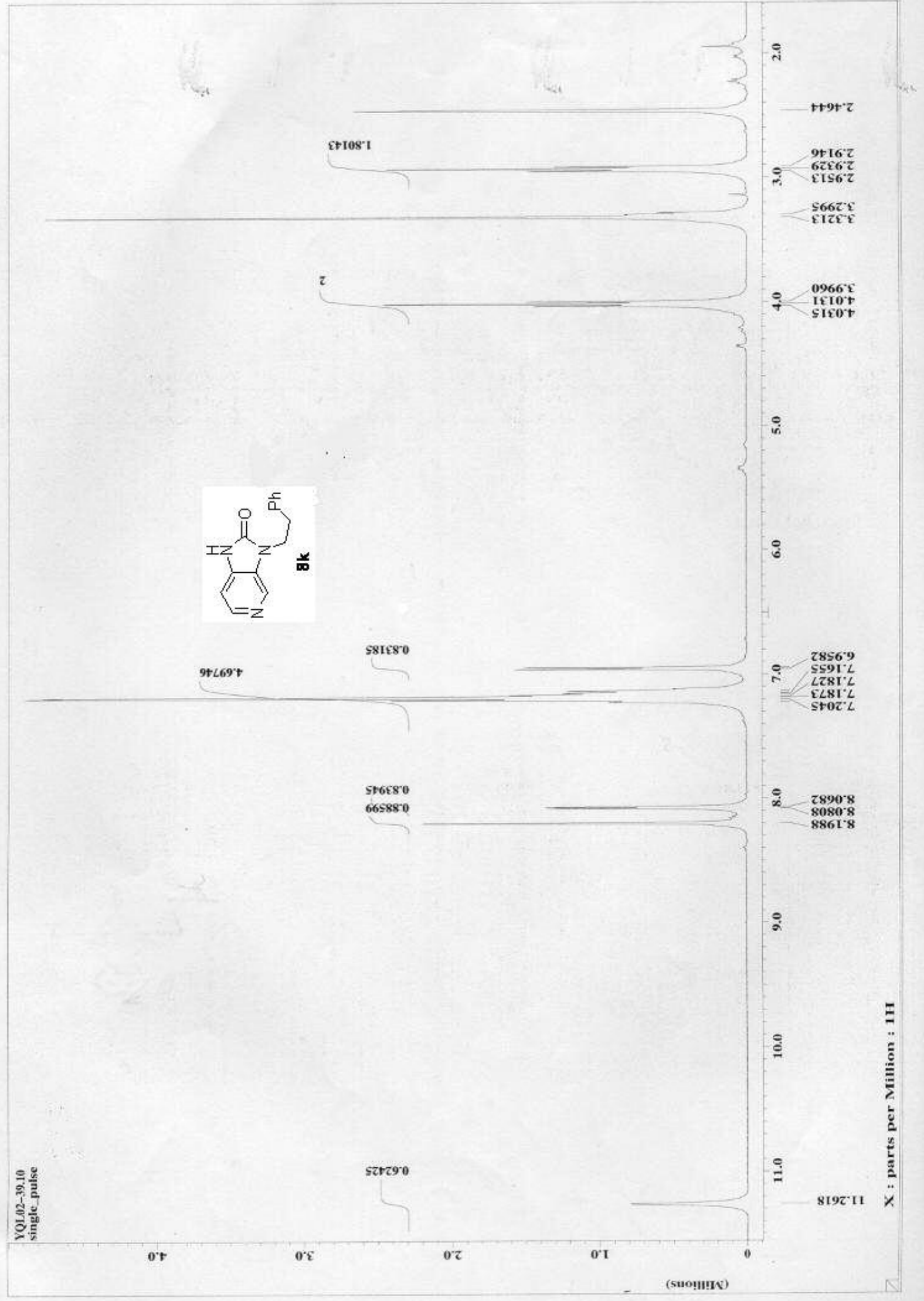




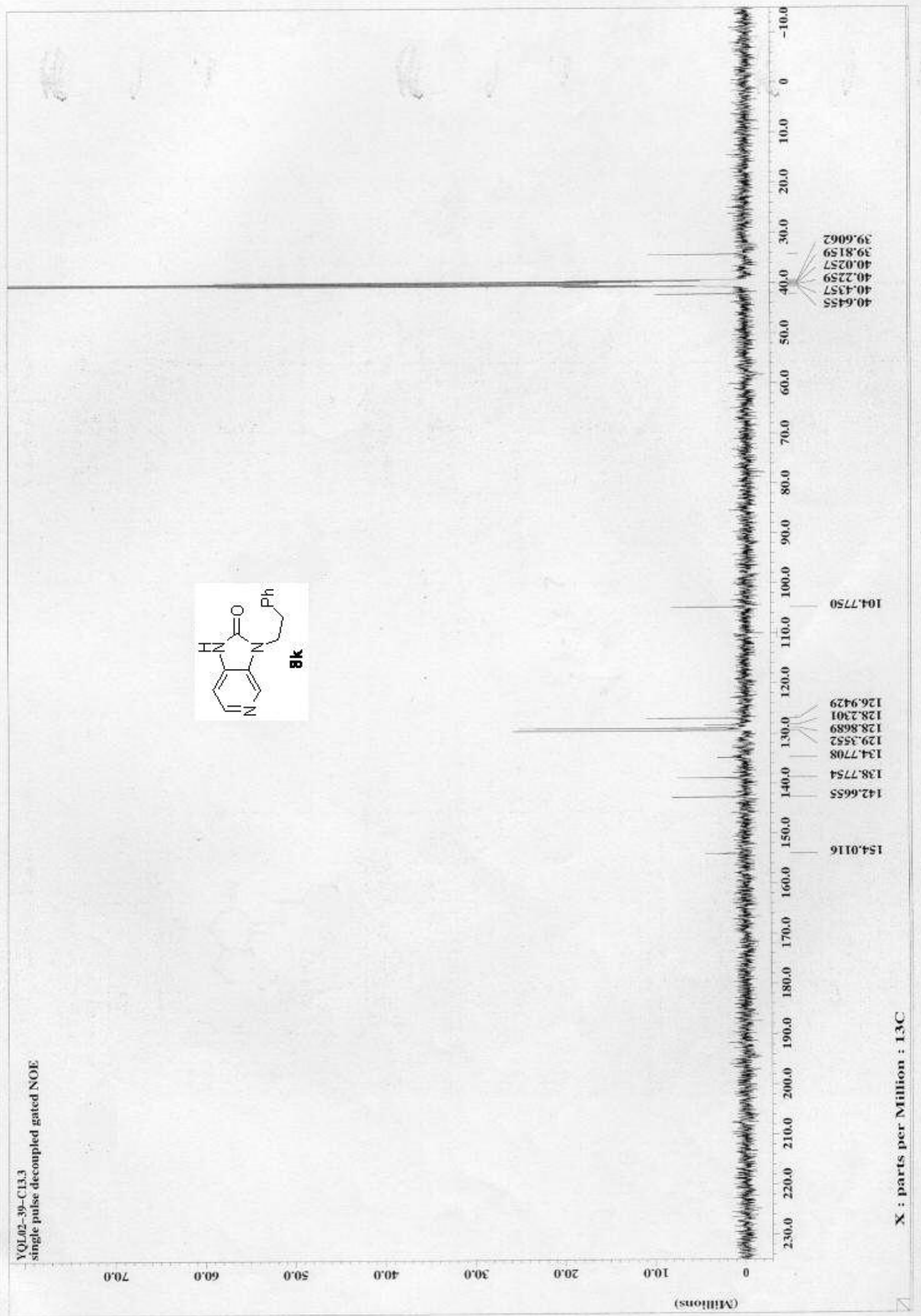









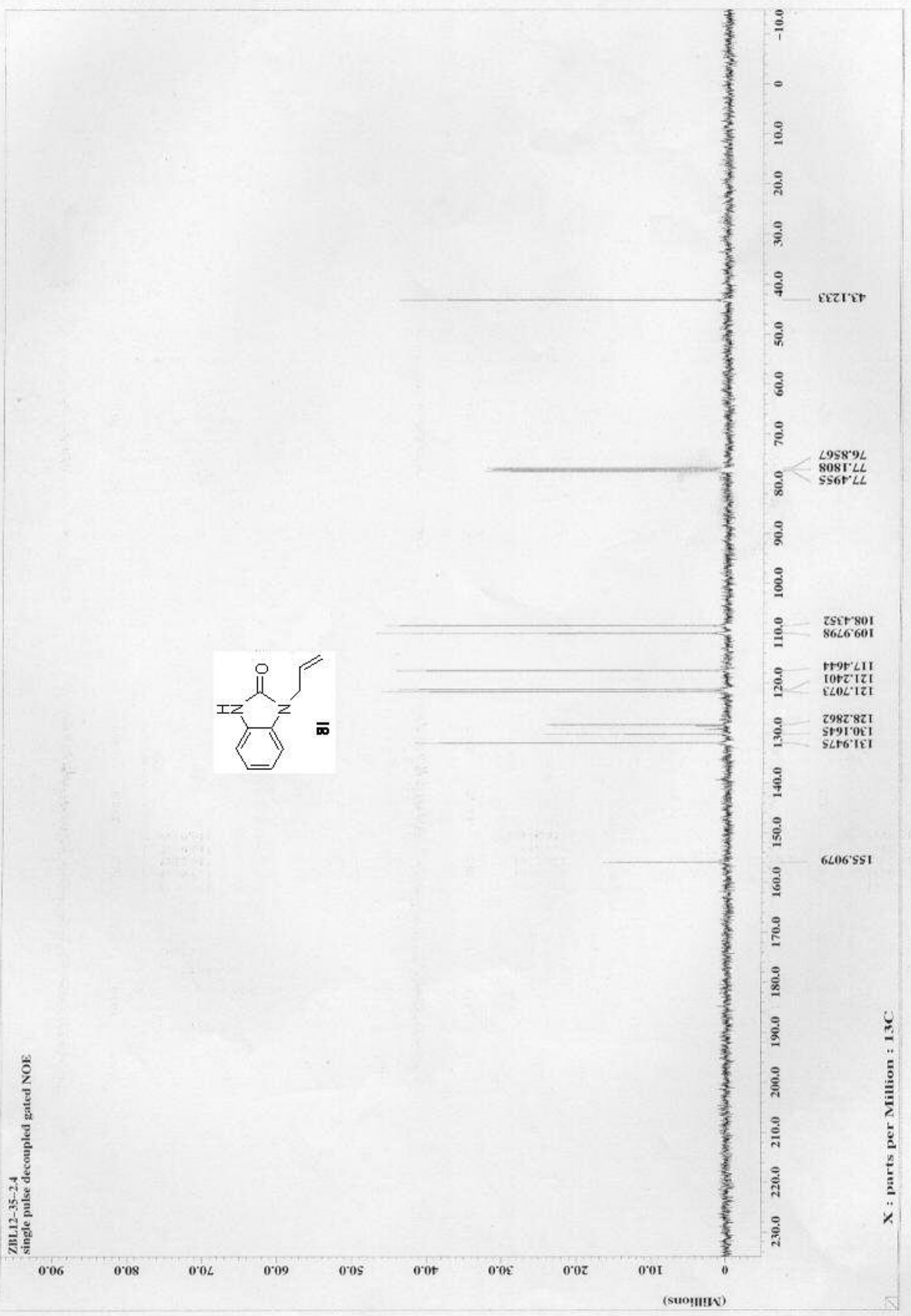




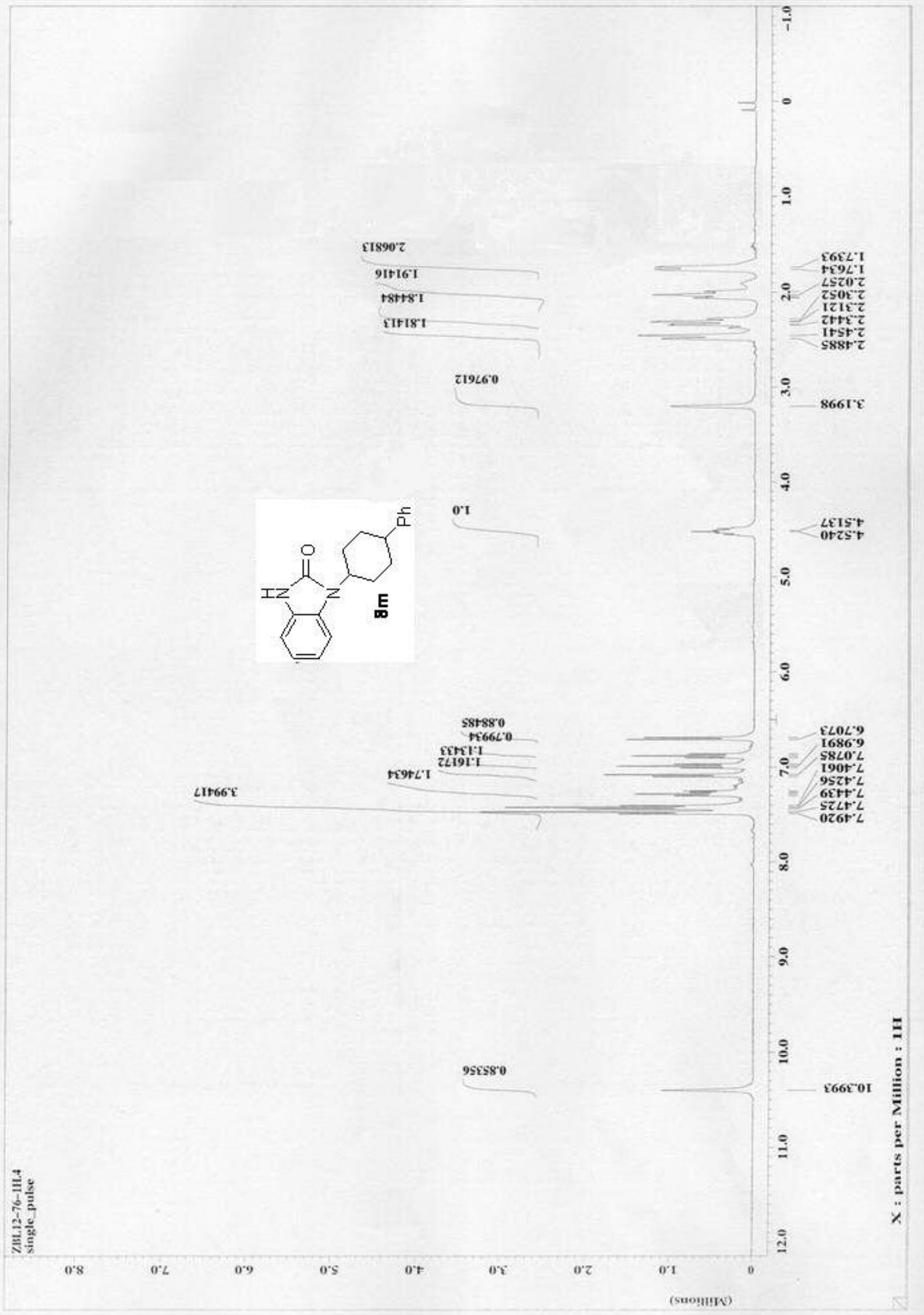




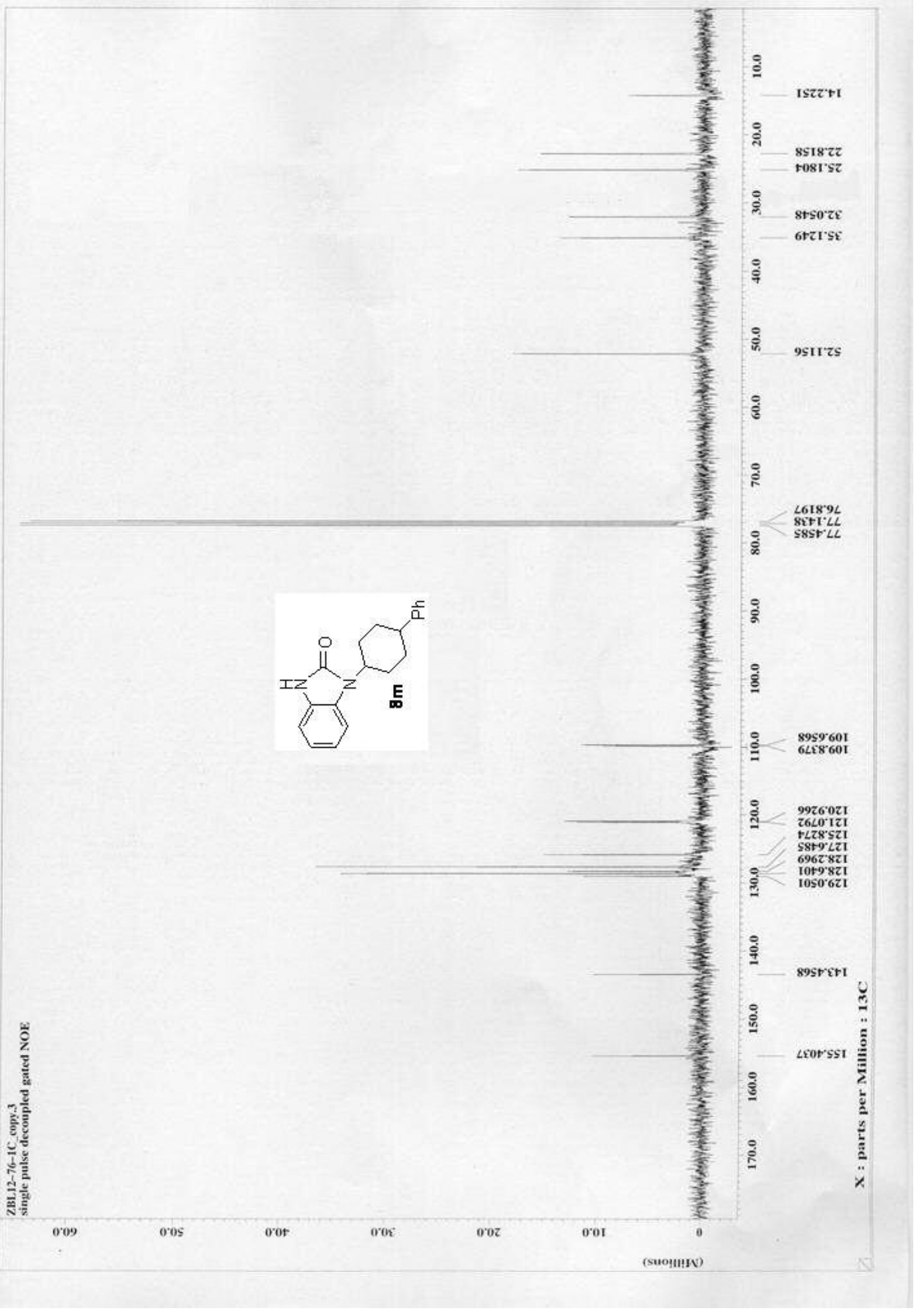




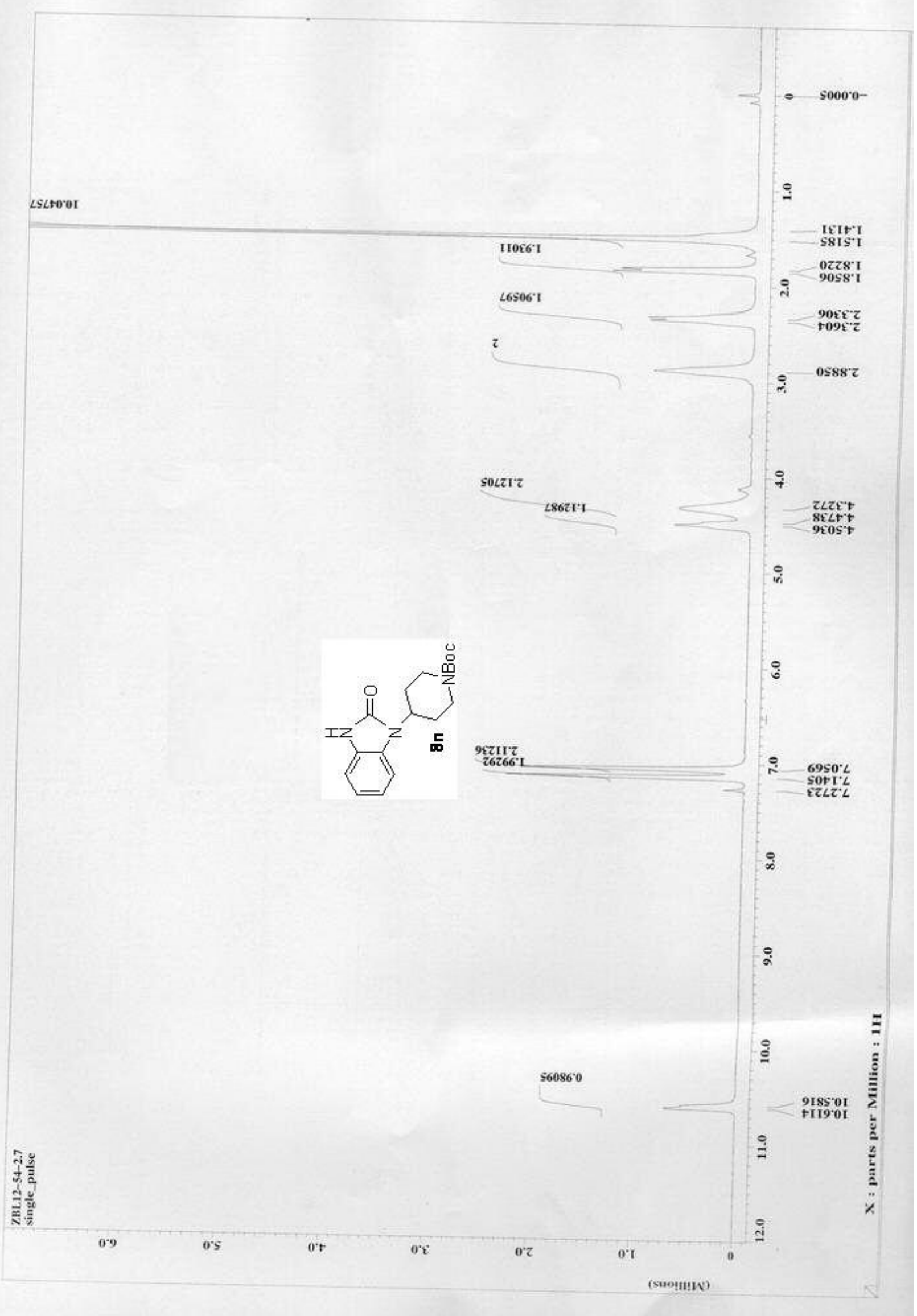




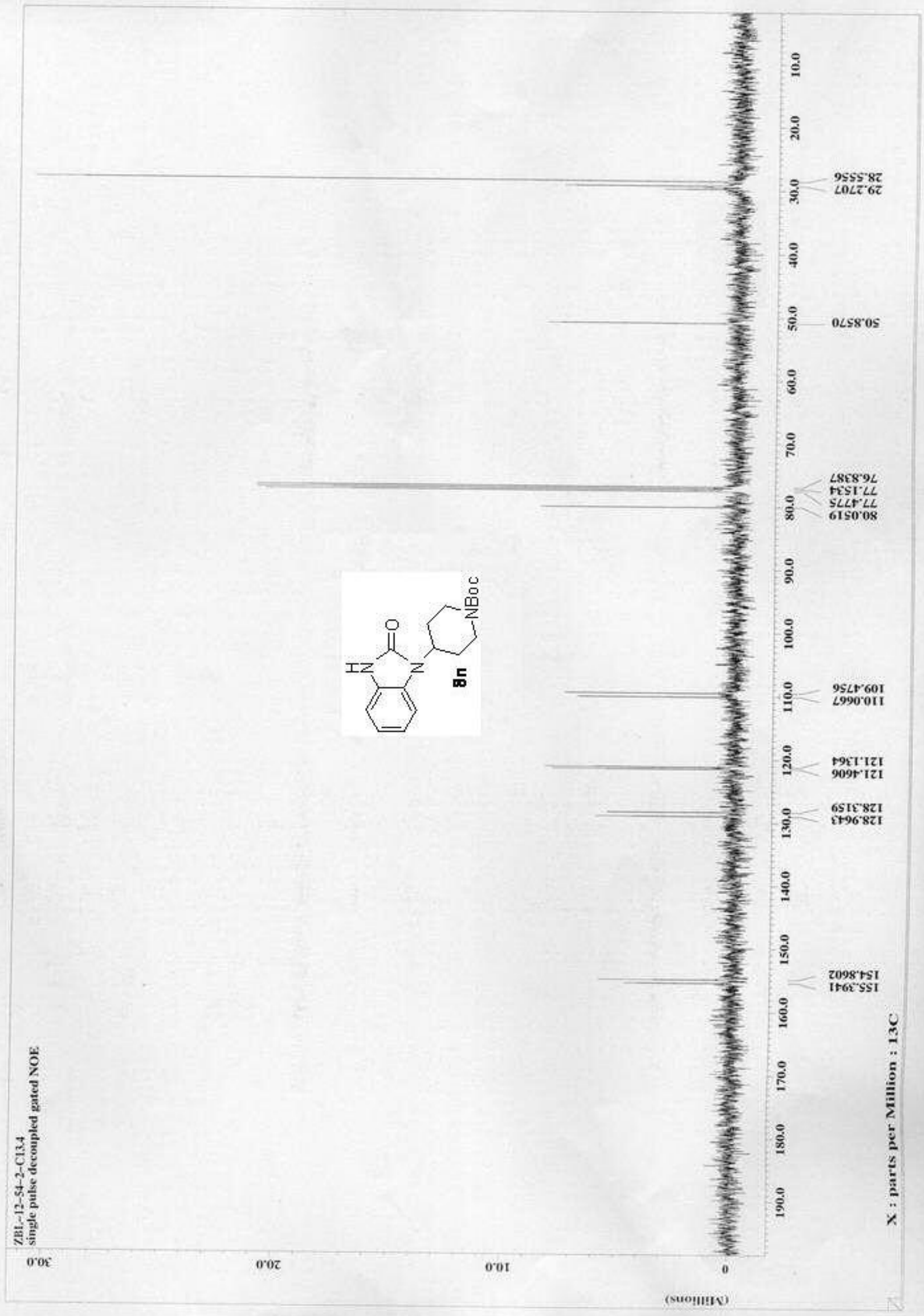




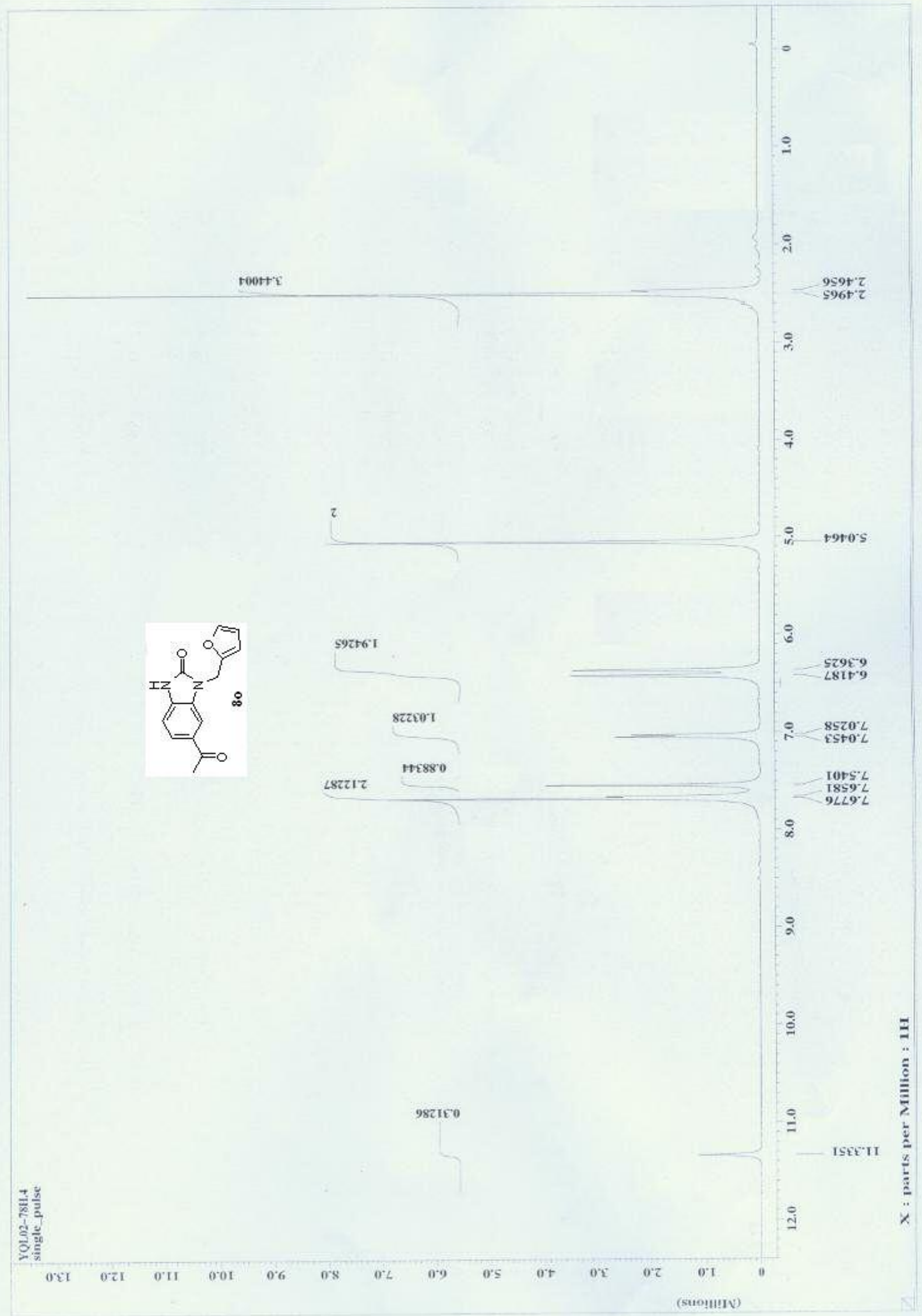




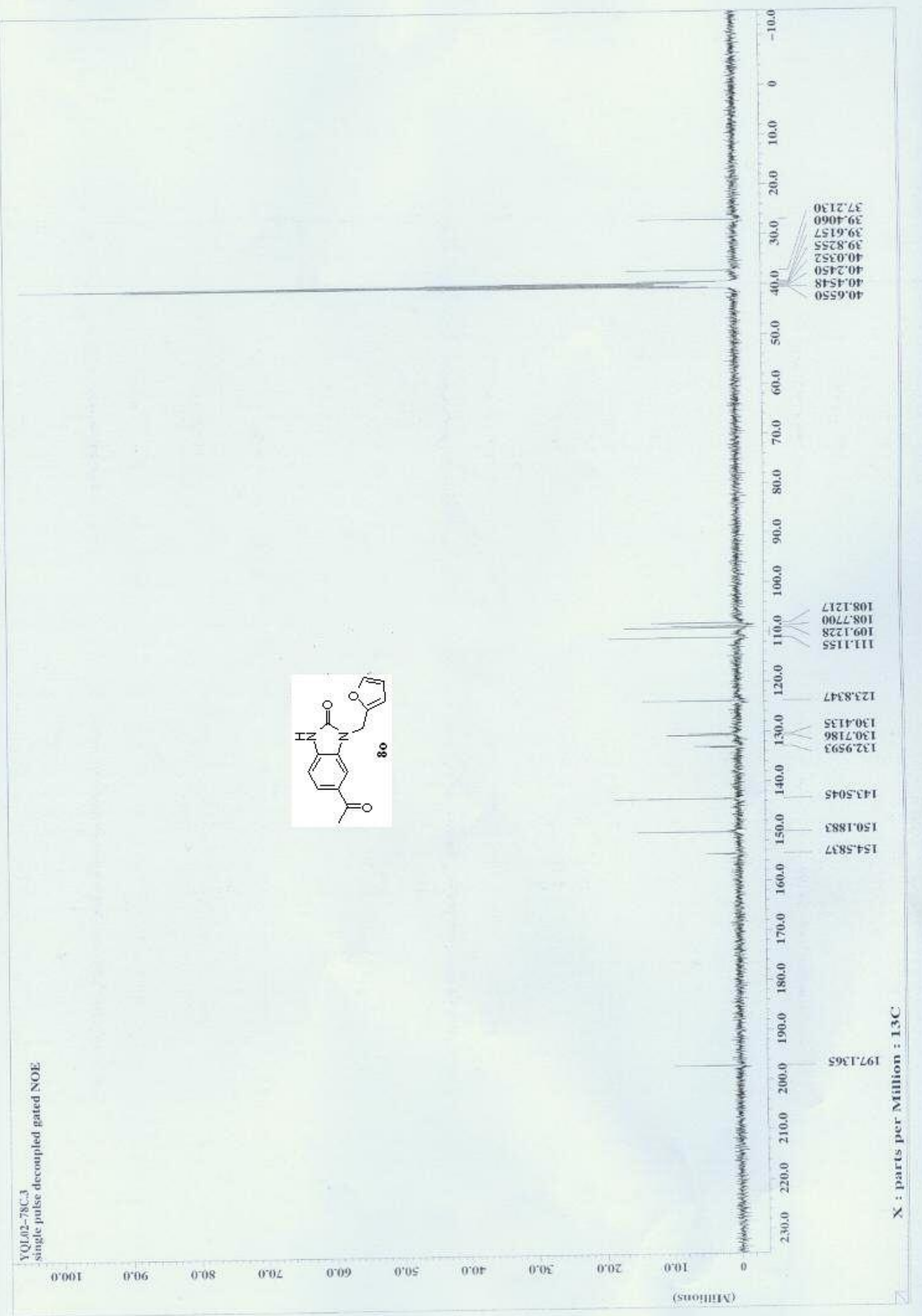




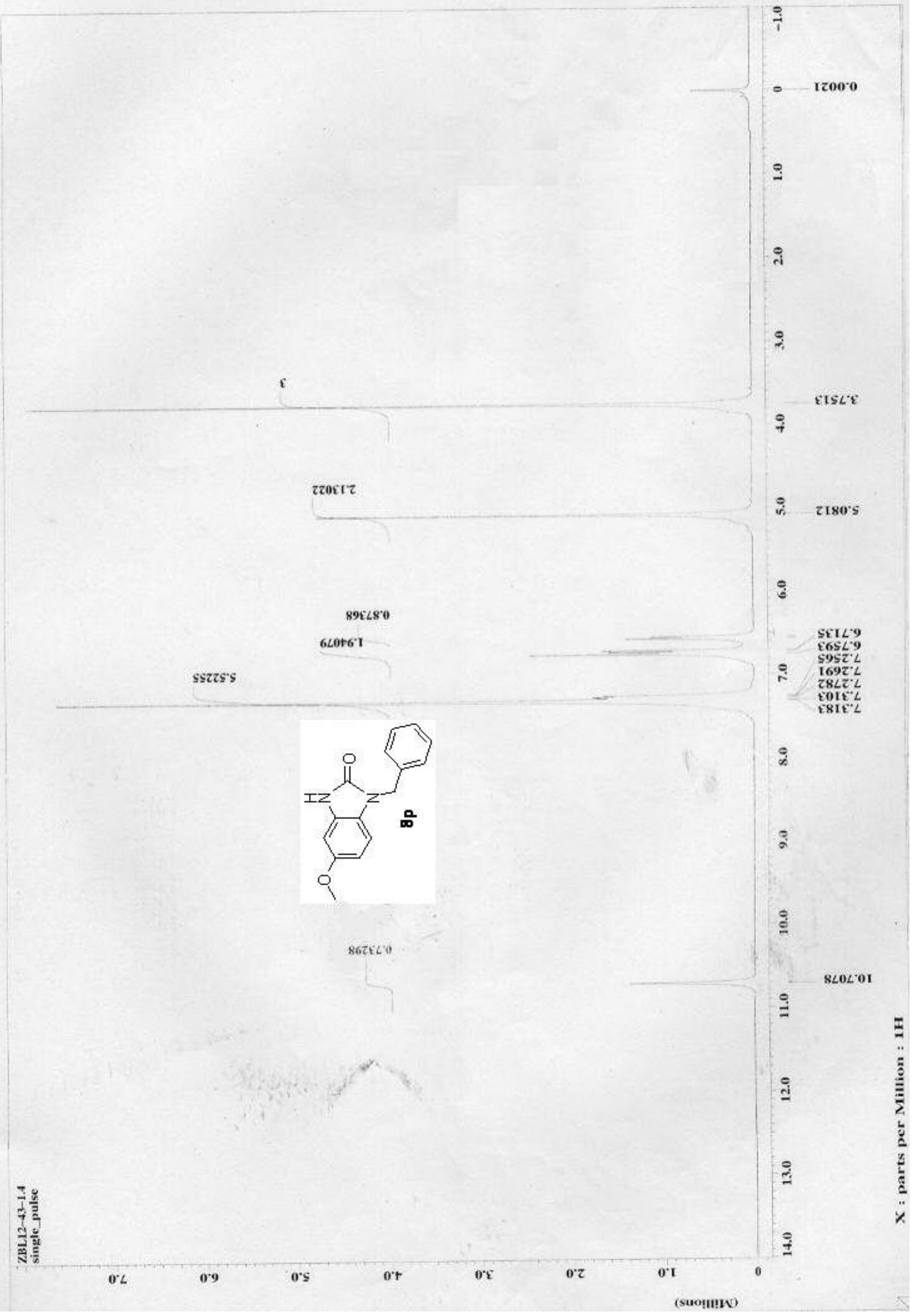




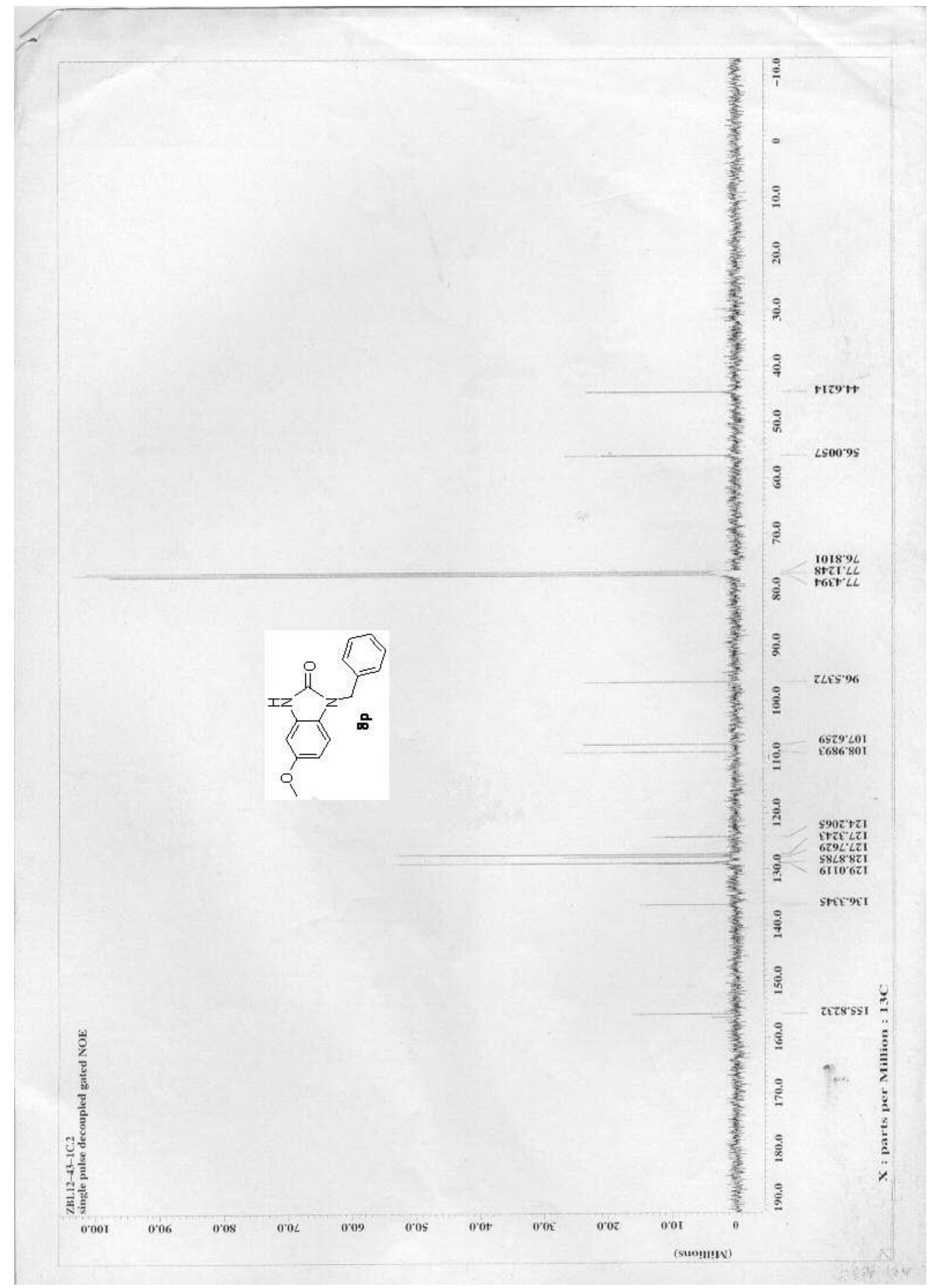




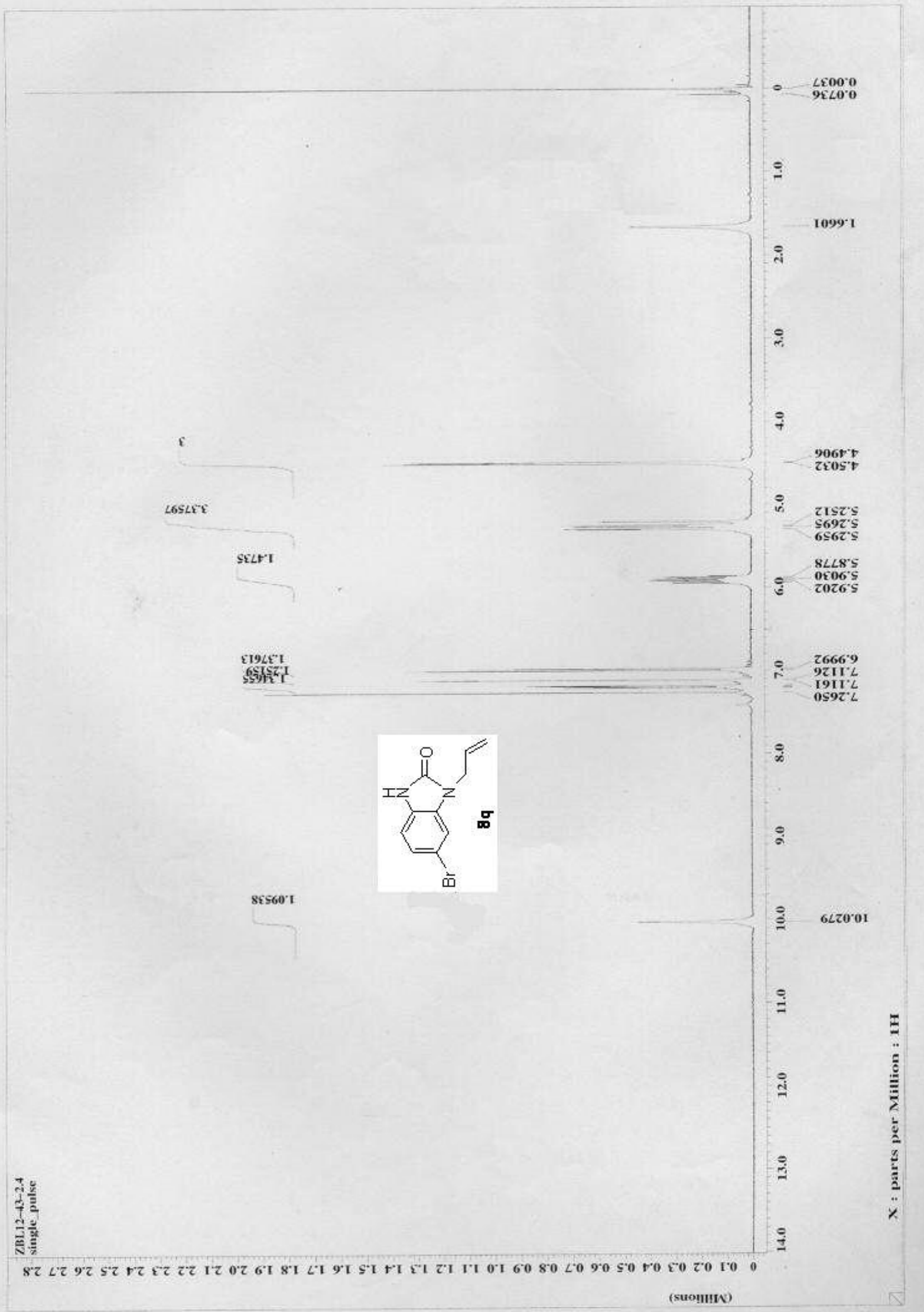




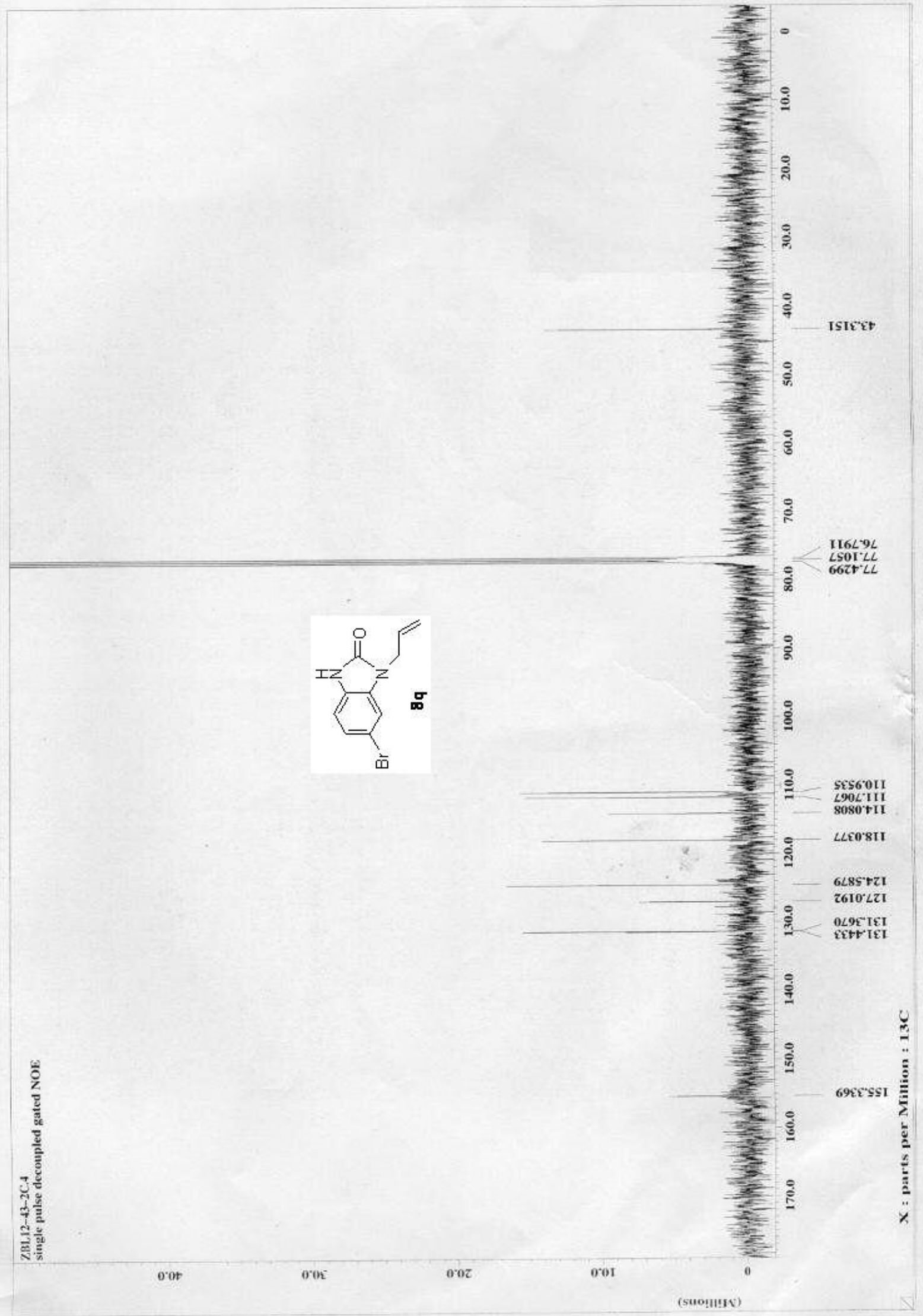

